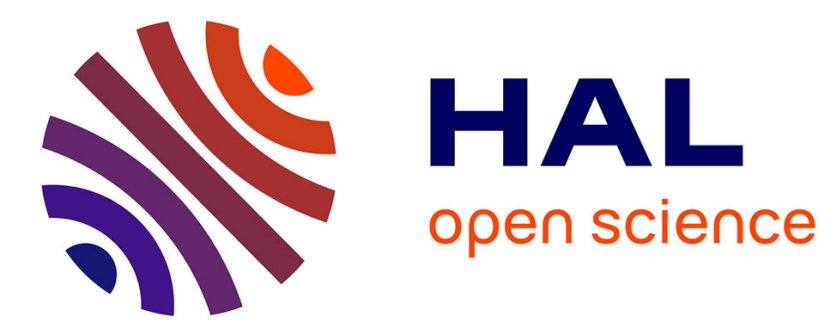

\title{
Shear-flow trapped-ion-mode interaction revisited. II. Intermittent transport associated with low-frequency zonal flow dynamics
}

\author{
Alain Ghizzo, F. Palermo
}

\section{To cite this version:}

Alain Ghizzo, F. Palermo. Shear-flow trapped-ion-mode interaction revisited. II. Intermittent transport associated with low-frequency zonal flow dynamics. Physics of Plasmas, 2015, 22 (8), pp.82304 82304. 10.1063/1.4928103 . hal-01285765

\section{HAL Id: hal-01285765 \\ https://hal.science/hal-01285765}

Submitted on 11 May 2018

HAL is a multi-disciplinary open access archive for the deposit and dissemination of scientific research documents, whether they are published or not. The documents may come from teaching and research institutions in France or abroad, or from public or private research centers.
L'archive ouverte pluridisciplinaire HAL, est destinée au dépôt et à la diffusion de documents scientifiques de niveau recherche, publiés ou non, émanant des établissements d'enseignement et de recherche français ou étrangers, des laboratoires publics ou privés. 


\section{Shear-flow trapped-ion-mode interaction revisited. II. Intermittent transport associated with low-frequency zonal flow dynamics}

A. Ghizzo, and F. Palermo

Citation: Physics of Plasmas 22, 082304 (2015); doi: 10.1063/1.4928103

View online: https://doi.org/10.1063/1.4928103

View Table of Contents: http://aip.scitation.org/toc/php/22/8

Published by the American Institute of Physics

\section{Articles you may be interested in}

Shear-flow trapped-ion-mode interaction revisited. I. Influence of low-frequency zonal flow on ion-temperaturegradient driven turbulence

Physics of Plasmas 22, 082303 (2015); 10.1063/1.4928102

Shear flow instabilities induced by trapped ion modes in collisionless temperature gradient turbulence

Physics of Plasmas 22, 042304 (2015); 10.1063/1.4916770

Spatio-temporal evolution of the $\mathrm{L} \rightarrow \mathrm{I} \rightarrow \mathrm{H}$ transition

Physics of Plasmas 19, 092306 (2012); 10.1063/1.4753931

Electron temperature gradient driven turbulence

Physics of Plasmas 7, 1904 (2000); 10.1063/1.874014

Influence of sheared poloidal rotation on edge turbulence

Physics of Fluids B: Plasma Physics 2, 1 (1990); 10.1063/1.859529

Zonal flow generation and its feedback on turbulence production in drift wave turbulence

Physics of Plasmas 20, 042304 (2013); 10.1063/1.4802187

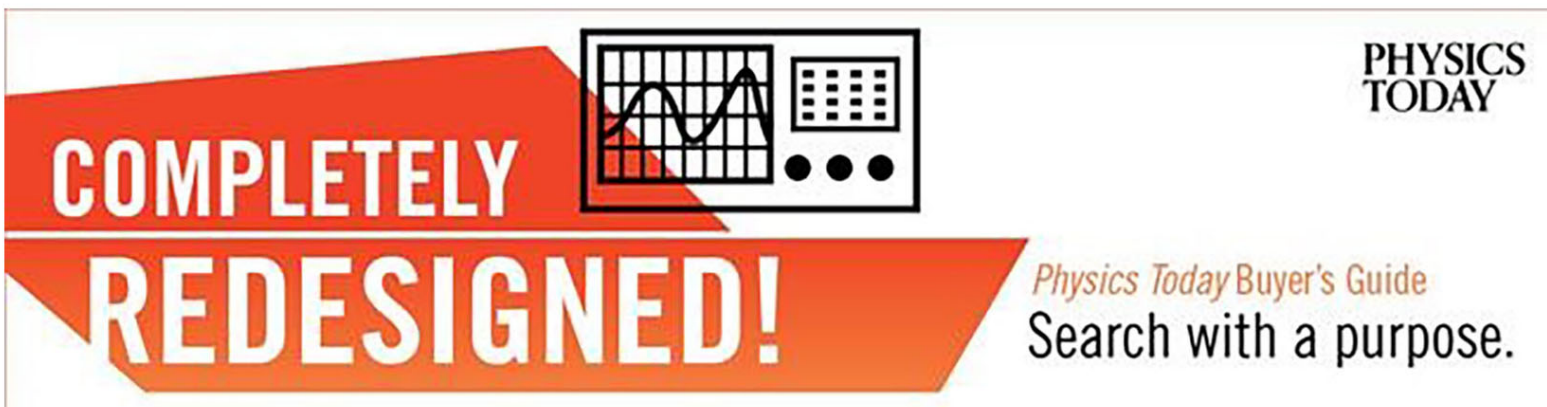




\title{
Shear-flow trapped-ion-mode interaction revisited. II. Intermittent transport associated with low-frequency zonal flow dynamics
}

\author{
A. Ghizzo ${ }^{1, a)}$ and F. Palermo ${ }^{2}$ \\ ${ }^{1}$ Institut Jean Lamour- UMR 7168, Université de Lorraine, BP 239 F-54506 Vandoeuvre les Nancy, France \\ ${ }^{2}$ LFMA -Ecole Centrale de Lyon, Université de Lyon, 60134 Ecully, France
}

(Received 3 April 2015; accepted 17 July 2015; published online 6 August 2015)

We address the mechanisms underlying low-frequency zonal flow generation in turbulent system and the associated intermittent regime of ion-temperature-gradient (ITG) turbulence. This model is in connection with the recent observation of quasi periodic zonal flow oscillation at a frequency close to $2 \mathrm{kHz}$, at the low-high transition, observed in the ASDEX Upgrade [Conway et al., Phys. Rev. Lett. 106, 065001 (2011)] and EAST tokamak [Xu et al., Phys. Rev. Lett 107, 125001 (2011)]. Turbulent bursts caused by the coupling of Kelvin-Helmholtz (KH) driven shear flows with trapped ion modes (TIMs) were investigated by means of reduced gyrokinetic simulations. It was found that ITG turbulence can be regulated by low-frequency meso-scale zonal flows driven by resonant collisionless trapped ion modes (CTIMs), through parametric-type scattering, a process in competition with the usual KH instability. @ 2015 AIP Publishing LLC. [http://dx.doi.org/10.1063/1.4928103]

\section{INTRODUCTION}

An important role of zonal flows (ZFs) in regulating turbulence and transport in tokamaks is now broadly accepted. $^{1,2}$ Most gyrokinetic numerical experiments (see the review paper in Ref. 3) highlight the crucial role of ZFs concentrated on ion temperature gradient (ITG) or trapped electron mode (TEM) turbulence. A classical paradigm for turbulent transport in tokamaks is drift wave (DW) turbulence, and the dynamics of a coupled system of DW and ZFs has been intensively studied. For both types of turbulence, three major governing processes are invoked: the generation of turbulence (for instance DW), self-organization of ZFs, and their damping or destabilization. The instabilities that lead to these changes correspond to changes in equilibrium solution $^{4,5}$ and/or the occurring of intermittence in turbulence in Refs. 6-8.

Zero-frequency ZFs are poloidal $\boldsymbol{E} \times \boldsymbol{B}$ plasma flows due to toroidally and poloidally symmetric $(m=n=0)$ potential perturbations. It is now well-known that ZFs can be damped by collisions. Such an approach is based on the collisional nature of the relaxation process. In particular, for very low collisional regime, it leads to a situation, where the characteristic time of relaxation is longer than the corresponding transit time $\left(\omega_{t}^{-1}\right)$ or bounce time $\left(\omega_{b}^{-1}\right)$ for trapped particles: $\partial_{t} \ll \omega_{t} ; \omega_{b}$. If this ordering cannot be applied and fast processes with $\partial_{t} \simeq \omega_{b}$ are allowed, then there is an additional branch, discovered by Winsor et al. in Ref. 9 referred usually as the geodesic acoustic mode (GAM) with a frequency $\omega_{G A M} \simeq \frac{v_{t h}}{R_{0}}$. GAMs have their own relaxation, sensitive to Landau resonance condition. ${ }^{10,11}$ Thus, highfrequency (HF) GAMs are damped by ion Landau resonance. Zero-frequency ZFs are, in turn, damped only by collisions on a longer time scale. Thus, GAMs introduce an alternate state dominated by oscillatory ZFs near the critical

\footnotetext{
a)alain.ghizzo@univ-lorraine.fr
}

temperature gradient and may lead to a new type of intermittent dynamics. Thus, plasma exhibits an intermittent dynamics, a process already observed in DW turbulence in Refs. 8 and 12 and trapped ion mode (TIM) turbulence in Ref. 13. Furthermore, a new intermittence was identified to originate from the collisionless damping of GAMs in zonal flow-TEM turbulence system in Refs. 14-16.

When toroidal effects, such as magnetic curvature and $\nabla B$ drifts, are incorporated in the model and when a proper kinetic treatment of trapped particles is required, the most prominent type of long wavelength toroidal micro-instability is collisionless TIMs in the presence of a significant ion temperature gradient. One of the most important features of these instabilities is their global nature. TIMs have a real frequency below the diamagnetic drift frequency $\omega_{* i}$ and below the ion bounce frequency $\omega_{b}$. These instabilities are easier to excite in the core region of tokamak fusion plasmas where the plasma can be considered to be effectively collisionless since $\omega_{* i}$ and $\omega_{b}$ are well above the ion-ion collision frequency. Hence, for the time scales of interest, collisions are not expected to have a significant effect on ZF damping. TIMs are a simple prototype of kinetic modes induced by the resonance of trapped ions with fluctuations through their precession motion. On the other hand, TIMs can also be considered as a prototype of interchange instability.

A major role in the complex interaction between $\mathrm{ZFs}$, TIMs, and nonlinear streamers is expected to be played by the Kelvin-Helmholtz $(\mathrm{KH})$ instability, as predicted by Refs. 17-20. Collisionless TIMs are known to be subject to interchange-type instabilities, their hybrid fluid-kinetic aspects reveal new features. In Paper I, we have found that in toroidal geometry, although the drive of the lowfrequency (LF) ZFs via Reynolds stress remains, the ZF structure results from the coupling with interchange-type TIMs, i.e., we have $\langle\phi\rangle_{Z F}=\langle\phi\rangle_{Z F, K H}+\langle\phi\rangle_{Z F, C T I M}$. In the previous equation, the first term is driven by the Reynolds tensor and linked to shear flow, while the second term is here 
driven by CTIM (interchange or/and resonant mode). Thus, the resulting ZF has a non-zero (low-frequency) real frequency close to the toroidal precession motion $\omega_{d 0} \bar{\omega}_{d}(\kappa)$ (here, $\bar{\omega}_{d}$ is a factor taking into account the different trapped ion populations by their respective pitch-angle parameter $\kappa$ ). One can think of the resulting ZF driven by CTIMs as a lowfrequency version of the GAM mode.

Recently, in Ref. 21, the authors have observed in numerical simulations that energy transfer can occur throughout the relaxation phase of the streamer growth, leading to $\mathrm{ZF}$ amplification when $\mathrm{KH}$ coupling takes place. We have observed that the energy is transferred from flows along the radial direction to flows oriented along the poloidal direction. Hence, it appears that the KH instability can play a major role not only as a mechanism of saturation of streamers but also as a mechanism of generation of convective cells, such as ZFs. Our major objective is to create an unified picture of the nonlinear interaction between TIMs, ZFs, KH modes, and nonlinearly generated streamers driven by the ITG instability. Our investigation has identified two significant mechanisms: first, a modification in the coupling between interchange-type CTIMs and secondly, the possible regulation of ITG turbulence by resonant ZF induced by three-wave parametric type instability. The remainder of this paper is organized as follows. The relation of these physical pictures to tokamak operating regimes is presented in Sec. II. In Sec. III, we study the coupling between the KH and the interchange instabilities using the Taylor-Goldstein formulation and we determine the different regimes of the coupling. The case of the parametric decay of an interchange mode will be also considered in this section when the CTIM becomes resonant with precessional trapped ions. In Section IV, we discuss the numerical results and give our conclusions in Section V.

\section{CONNECTIONS WITH EXPERIMENTAL SITUATIONS}

Experimental papers reported various appearances of both high-frequency GAM and low-frequency ZF oscillations. Such oscillations are found recently only in limited regions of the plasma in tokamak experiments, such as the DIIID tokamak, ${ }^{22}$ the ASDEX Upgrade experiment in Ref. 23, or the EAST tokamak in Ref. 24. ZFs have been broadly classified into two branches, one being a zero-frequency ZF (as stated in the hydrodynamical theory) and the second being an oscillating flow at high frequency and termed the geodesic acoustic mode due to the complex magnetic topology met in tokamaks. The former results in the fluid framework from the Reynolds stress, the latter is thought to be driven by nonlinear interactions with turbulence through three-wave coupling. High-frequency GAM is strongly (Landau) damped in the core, where the zonal flow is thought to be more prevalent, and may only exist towards the tokamak's edge, where it is weakly damped at high values of the magnetic safety factor. GAMs are not typically observed in the H-mode plasma. From a physical point of view, GAM can be considered as ion acoustic wave (IAW) in the geodesic version due to the magnetic geometry of the tokamak. Indeed, GAMs degenerate to the standard IAWs in the cylindrical geometry.

On the experimental side, some of the defining GAM characteristics have been observed, including the occurring of a low-frequency ZF counterpart. In the DIIID tokamak (see Ref. 22) in addition of GAM, a low-frequency broadband feature was also observed indicating the presence of a low-frequency fluctuations with peak located at $4 \mathrm{kHz}$ in the core of tokamak. The amplitude of such low-frequency ZF was seen to increase with decreasing $\frac{r}{a}$, while the GAM intensity appears to decrease. More recently, oscillations, of electrostatic nature, were observed in the plasma edge at much lower frequency than the GAM in the EAST tokamak experiment (see Ref. 24 for more details). The fluctuating potential power spectra exhibit a peak close to $2 \mathrm{kHz}$ with two harmonics and with background peaking at $80 \mathrm{kHz}$, while GAM does not appear to be active in the Low-High (LH) transition process under these experimental conditions. It was also reported that the frequency spectrum may exhibit more intermittent features. The existence of three-wave coupling between the turbulence in $80 \mathrm{kHz}$ and the lowfrequency $2 \mathrm{kHz}$ oscillation was also reported in this experiment. Similar behaviour was also reported in the ASDEXUpgrade tokamak in Ref. 23.

However, the nature of GAMs can also be modified by fast ions, leading to a new branch, the so-called EGAM for energetic particle induced GAM. The corresponding upward frequency chirping is attributed to the formation of holeclump pair in phase space (see Ref. 25). The downward frequency chirping is also possible in principle. Since the pioneering work of Bernstein, Greene, and Kruskal (BGK) in 1957 in Ref. 26, it is well-known that such phase space holes are self-sustained and connected to electrostatic fields that are self-consistent with some manner of trapped particle velocity distribution function. Recently, laser-plasma interaction experiments revealed that such electron trapping structures might conceivably be physically relevant thus leading to renewed interest in the subject. Indeed, there is a similarity between the recent observations of low-frequency ZFs and some recent experimental results obtained in the laser-plasma interaction in the so-called kinetic regime of the interaction. A clear indication of the kinetic behaviour induced by electrostatic trapping was the recent observations in Ref. 27, at the trident laser facility of stimulated Raman scattering (SRS), associated with what was termed Stimulated Electron Acoustic Scattering (SEAS), a novel scattering apparently involving a so-called Electron Acoustic Wave (EAW), whose phase space velocity $v_{\varphi} \simeq 1.3 k v_{t h}$ is between an electron plasma wave (EPW) and an IAW $\left(v_{\text {th }}\right.$ being the electron thermal velocity). The experimental results show a small narrow signal with an uncharacteristic frequency downward shift of about $0.37 \omega_{p}\left(\omega_{p}\right.$ being the plasma frequency) which could not be the usual plasma (high frequency) SRS (also seen but at much higher levels $\sim 3000$ times stronger) with its frequency shift at about $\omega_{p}$. Such EAWs are justified in the limit of small amplitude waves, which depends essentially from the population of trapped electrons. The concept of these waves is indeed a novel version of the BGK nonlinear waves with electrons 
trapped in the wave troughs. Note that it is the fact that such EAWs may back react with the plasma (in particular, in a diffusion process with existing electromagnetic waves) which make possible their detection in experimental situations. Motivated by the Montgomery's experiment in laserplasma experiment, Johnston et al. in Ref. 28 carried out numerical experiments using Vlasov-Poisson model and observed states, referred as kinetic electrostatic electron nonlinear (KEEN) waves. Ponderomotively driven, KEEN waves are seen to self-consistently form and persist at nearly constant amplitude long after the driver is turned off. We note that in a separate research thread in Ref. 29, KEEN waves are also generated in Vlasov-Maxwell simulations relevant to SRS scenario.

The situation observed in the EAST experiment resembles the case of an ion geodesic version of low-frequency EAW, while the role of high-frequency EPW is here played by GAM (see Table I in which we have summarized the different processes). Our investigation reveals that it is the magnetic particle trapping (and the resulting population of trapped ions through the CTIM and/or CTEM for collisionless trapped electron modes) which determines the resonance conditions of the residual low-frequency GAM-type zonal flow. Thus, here a key physical point is that low-frequency $\mathrm{ZF}$ excitation is due to strong magnetic trapping (even if the population of trapped particles as in the Montgomery's laserplasma experiment is weak) and the resulting resonances with precessing trapped ions. As a consequence, the mode is expected to be observed when the others standard modes as GAMs and zero-frequency ZFs remain at a very weak level, a situation met in the EAST or ASDEX-Upgrade experiments in the $\mathrm{H}$ mode regime in the plasma edge. Note that in principle, the mode can also be observed in the plasma core as in the DIII-D experiment in Ref. 22 but both ZFs coexist (oscillating plus zero-frequency fluid modes).

The last point concerns the possible coupling of such a low-frequency (kinetic) ZF mode with the plasma environment. Recent experiments, conducted at the TEXTOR tokamak, in biasing $\mathrm{H}$-mode regime in Ref. 30, have shown intimate interaction among sheared flows, Reynolds stress, and ZFs across a transition to improved confinement. Here, the coupling with the interchange-type turbulence is allowed by the introduction of the mean shear flow term through the Kelvin-Helmholtz instability. Note that a similar approach is proposed in Ref. 31 for describing recent experimental observations of the LH transition with shear flow driventurbulence in DIII-D.

\section{CTIM-KH COUPLING}

We first consider processes involving the origin and evolution of ZFs with a particular emphasis on the influence of the KH instability on ZF stability. The coupling of TIMs and $\mathrm{KH}$ modes has been considered in the context of the Taylor-Goldstein formulation, which reads as

$$
\begin{gathered}
\varphi^{\prime \prime}-\left(k^{2}-\frac{\omega_{0}^{\prime \prime}}{C-\omega_{0}}\right) \varphi-\frac{C_{e}\left(\omega_{0}-\frac{d\langle\phi\rangle_{\alpha}}{d \psi}\right)}{C_{i} \delta_{b}^{2}\left(C-\omega_{0}\right)} \varphi \\
-\frac{3}{2} \frac{\omega_{d 0} P_{0}^{\prime}(\psi) \varphi}{C_{i} \delta_{b}^{2}\left(C-\omega_{0}\right)^{2}}=0,
\end{gathered}
$$

where $\varphi=\varphi(\psi)$ denotes the electric potential fluctuation of mode $n, k^{2}=\frac{C_{e}}{C_{i} \delta_{b}^{2}}+\frac{n^{2} \rho_{s}^{2}}{\delta_{b}^{2}}$, and $C=\frac{\omega}{n}$. While the second term in Eq. (1) corresponds to the usual KH eigenmode, the fact to take into account TIMs leads to the second and third terms related to the interchange turbulence. Note that in Eq. (1), the prime corresponds to the derivation with respect to $\psi$ and $\omega_{0}(\psi)=\phi_{0}^{\prime}$ describes the generalized shear flow. Details were given in Appendix A.

Equation (1) is known as the Taylor-Goldstein equation, which is used to study stratified shear flow instabilities occurring in a variety of physical contexts, such as astrophysics, the Earth's atmosphere or oceanography. In particular, in stratified shear flows, the class of unstable modes can be further divided into two subclasses of unstable modes, those whose phase velocity is zero with respect to mean flow (i.e., $\mathrm{KH}$ modes) and those whose phase velocity is nonzero, the Holmboe modes in Refs. 20, 32, and 33. In particular, for such systems, it was shown in Ref. 34, in shear flow with high Reynolds numbers, that the wave-flow resonance plays a key role in the interaction, where the phase velocity is different from zero. In plasmas, the wave-flow resonance is produced by the resonant character of CTIMs in the collisionless regime, which can propagate in the ion diamagnetic direction. This is in contrast to the standard picture of (dissipative) TIMs, introduced by Kadomtsev and Pogutse in Ref. 35, which propagate in the electron diamagnetic

\begin{tabular}{|c|c|c|}
\hline & Tokamak's experiment & Laser-plasma experiment \\
\hline HF mode & $\begin{array}{c}\text { GAMs observed in the edge } \\
\text { plasma (geodesic version of IAWs) }\end{array}$ & $\begin{array}{c}\text { EPWs (observed in backward } \\
\text { Raman scattering) }\end{array}$ \\
\hline LF mode & $\begin{array}{c}\text { Oscillating zonal flow } \\
\text { induced by magnetic trapping }\end{array}$ & EAWs induced by electrostatic trapping \\
\hline Intermittent behaviour & Turbulence bursts & Bursty events in reflectivity \\
\hline Coupling mechanisms & $\begin{array}{l}\text { Three-wave process with } \\
\text { HF interchange modes and/or } \\
\text { KH instability Reynolds tensor }\end{array}$ & $\begin{array}{l}\text { SEAS for stimulated EAW scattering } \\
\text { coupling with laser waves }\end{array}$ \\
\hline Granulation effects & Streamers, zero-frequency (fluid) ZFs & EAWs (BGK waves) \\
\hline $\begin{array}{l}\text { Nonlinear coherent } \\
\text { structures }\end{array}$ & LF (kinetic) ZFs, generalized $\mathrm{KH}$ vortices & \\
\hline
\end{tabular}

TABLE I. Summary of different types of nonlinear waves and coupling processes in both magnetic and inertial confinement fusion devices. 
direction and are thus unable to resonate with precession (trapped) ions.

To prepare numerical simulations taking into account the coupling between ITG and KH instabilities, we discuss now on the choice of the initial distribution to be implemented in the Vlasov solvers. Before going to the analysis of the TIM/KH coupling, we briefly review the results in the two limits of pure interchange (i.e., collisionless TIM) and pure $\mathrm{KH}$ regimes.

\section{A. Collisionless trapped-ion mode regime}

TIMs are unstable when $\omega_{* i} \omega_{d 0} \sim \nabla P . \nabla B>0$ and so are analogous to interchange-type instability. Here, ITG is thus supported by trapped ions. However, we choose to start with an initial shear potential of $\phi_{0}(\psi)=\frac{3}{2} \omega_{d 0}\left\langle\bar{\omega}_{d}(\kappa ; s)\right\rangle_{\kappa} \psi$ $\simeq \frac{3}{2} \omega_{d 0} \psi$ assuming that $\kappa \simeq 0$ (deeply trapped ion regime) and therefore $\bar{\omega}_{d}=1$. A convenient choice of this class of TIM equilibrium is provided by

$$
F_{e q}=e^{-\frac{E}{T_{0}}}\left(1+\left(\frac{E}{T_{0}}-\frac{3}{2}\right) \omega_{d 0} \psi \Delta \tau\right) .
$$

Such choice corresponds to the data of charge density $\rho_{0}(\psi)=n_{i, e q}(\psi)-n_{0}=0$ and to an equilibrium pressure of $P_{0}(\psi)=n_{0} T_{0}\left(1+\omega_{d 0} \psi \Delta \tau\right)$. Thus, in Eq. (1) we can take $P_{0}^{\prime}(\psi)=\omega_{d 0} \Delta \psi$ and a "shear flow frequency" $\omega_{0}(\psi)=\frac{d \phi_{0}}{d \psi}$ $=\frac{3}{2} \omega_{d 0}$, i.e., $\omega_{0}^{\prime \prime}=0$ (no KH mode) and now Eq. (1) reads

$$
\left(\frac{\omega}{n}-\omega_{0}(\psi)\right)^{2}\left(\varphi^{\prime \prime}-k^{2} \varphi\right)=\frac{3}{2} \frac{\omega_{d 0}^{2} \Delta \tau}{C_{i} \delta_{b}^{2}} \varphi,
$$

where $k^{2}$ is given by the quantity $\frac{C_{e}}{C_{i} \delta_{b}^{2}}+\frac{n^{2} \rho_{s}^{2}}{\delta_{b}^{2}}$ and $\triangle \tau$ is the ion constant temperature gradient. Here, $\varphi=\delta \phi_{n}(\psi)$ corresponds to the potential fluctuation of mode $n$. We recall that the eigenfunctions are determined by $\varphi_{s}=\sin (l \pi \psi)$. By introducing $\omega=\frac{3}{2} n \omega_{d 0}+i \gamma$ and $\varphi_{s}^{\prime \prime}=-l^{2} \pi^{2} \varphi_{s}$ in Eq. (3), we obtain an estimation of the growth rate as follows:

$$
\frac{\gamma}{n}=\sqrt{\frac{3}{2}} \frac{\omega_{d 0} \sqrt{\triangle \tau-\Delta \tau_{\text {threshold }}}}{\sqrt{C_{i} \delta_{b}^{2} l^{2} \pi^{2}+C_{e}+C_{i} n^{2} \rho_{s}^{2}}} .
$$

Thus, the linear analysis of ITG leads to the occurring of a threshold in the ion temperature gradient (close to $C_{e}$ when neglecting the effects of the gyro-average operator). It must be pointed out that usually the interchange-type mode for TIM has a frequency close to $\omega=\frac{5}{2} n \omega_{d 0}$ (while the resonant counterpart of CTIMs is slightly weaker close to $\left.\frac{3}{2} n \omega_{d 0}\right)$.

\section{B. Dominant Kelvin-Helmholtz regime}

In the limit where $P_{0}^{\prime}(\psi) \rightarrow 0$, we recover the Rayleightype eigenvalue equation

$$
\begin{aligned}
& \left(C-\omega_{0}(\psi)\right)\left(\varphi^{\prime \prime}-k^{2} \varphi\right)-\frac{C_{e}}{C_{i} \delta_{b}^{2}}\left(\omega_{0}-\frac{d\langle\phi\rangle_{\alpha}}{d \psi}\right) \varphi \\
& +\omega_{0}^{\prime \prime}(\psi) \varphi=0 .
\end{aligned}
$$

The well-known Rayleigh's inflection point theorem requires the existence of an inflection point for the instability (see Ref. 36). However, it must be pointed out that the Rayleigh equation is here modified by the presence of the mean flow $\langle\phi\rangle_{\alpha}$ which must be taken into account since we have kept the factor $\omega_{d 0} \bar{\omega}_{d}(\kappa ; s) E \frac{\partial f}{\partial \alpha}$ in the Vlasov equation in the trapped ion model (Eq. (3) of Paper I). Thus, a necessary condition for Eq. (5) to have an unstable growing solution (i.e., a solution with $C$ complex) is that the flow $\omega_{0}(\psi)=\phi_{0}^{\prime}(\psi)$ must have an inflection point $\left(\omega_{0}^{\prime \prime}\left(\psi_{s}\right)=0\right)$ at a point $\psi_{s}$, somewhere in the domain where the flow is defined. A more general view of the effects of shear flow can be obtained by taking a simple case limit ( $\mathrm{KH}$ being dominant) which allows an analytic solution. Preliminary results for zero boundary conditions (i.e., $\varphi=0$ at the boundaries) have been reported in Paper I. In the present work, we complement those results. We choose here a dimensionless quantity $\frac{\phi_{0}(\psi)}{\omega_{d 0}}=\Delta \psi \frac{\sin (2 \pi \psi)}{2 \pi}+\left\langle\bar{\omega}_{d}(\kappa) E\right\rangle_{\kappa, E} \psi$, which corresponds to $\omega_{0}(\psi)=\omega_{d 0} \cos (2 \pi \psi)+\omega_{d 0}\left\langle\bar{\omega}_{d}(\kappa) E\right\rangle_{\kappa, E}$ in presence of a TIM shear flow defined by $\langle\phi\rangle_{\alpha}=\left\langle\omega_{d 0} \bar{\omega}_{k}(\kappa ; s) E\right\rangle_{\kappa, E} \psi$ (noted that its second derivative with respect to $\psi$ is chosen to be zero).

The equilibrium condition, previously given by Eq. (2) must be modified to describe now the $\mathrm{KH}$ regime. We choose here

$$
F_{e q}(\psi)=e^{-\frac{E}{T_{0}}}\left(1+\left(\frac{5}{2}-\frac{E}{T_{0}}\right) C_{i} \delta_{b}^{2} 2 \pi \sin (2 \pi \psi)\right),
$$

which allows us to define, in a self-consistent way, the initial data for density and pressure at the equilibrium. Thus, we obtain $\rho_{0}(\psi)=2 \pi C_{i} \delta_{b}^{2} \sin (2 \pi \psi)$ and $P_{0}(\psi)=n_{0} T_{0}$. Let us introduce the variable change $x=2 \pi \psi$. Equation (5) becomes then, where $\omega_{0}(x)=\omega_{d 0} \cos x+\omega_{d 0}\left\langle\bar{\omega}_{d}(\kappa) E\right\rangle_{\kappa, E}$,

$$
\begin{aligned}
& \left(C-\omega_{0}(x)\right)\left(\varphi^{\prime \prime}(x)-k^{2} \varphi\right) \\
& -\frac{C_{e}}{C_{i} \delta_{b}^{2} 4 \pi^{2}}\left(\omega_{0}-\frac{d\langle\phi\rangle_{\alpha}}{d x}\right) \varphi+\omega_{0}^{\prime \prime}(x) \varphi=0,
\end{aligned}
$$

where we have introduced the quantity $k^{2}=\frac{C_{e}}{C_{i} \delta_{b}^{2} 4 \pi^{2}}+\frac{n^{2} \rho_{s}^{2}}{4 \pi^{2} \delta_{b}^{2}}$ and where the prime notation refers now to the derivative with respect to $\mathrm{x}$.

The inflection points with $\omega_{0}^{\prime \prime}(x)=0$ are located at $x_{s}=\frac{\pi}{2}, \frac{3 \pi}{2}$. To find the neutrally stable solution (which we denotes by $\left.\varphi_{s}\right)$, we substitute $C=\left.\frac{\omega}{n}\right|_{n=n_{s}}=\left\langle\bar{\omega}_{d}(\kappa) E\right\rangle_{\kappa, E}=$ const and Eq. (7) becomes

$$
\varphi_{s}^{\prime \prime}(x)+\left(1+\frac{C_{e}}{C_{i} \delta_{b}^{2} 4 \pi^{2}}-k^{2}\right) \varphi_{s}(x)=0 .
$$

Equation (8) has been complemented by the proper boundary condition $\varphi_{s}=0$. The solution again writes $\varphi_{s}(x)=\sin \left(\frac{l x}{2}\right)$ with $\frac{n^{2} \rho_{s}^{2}}{4 \pi^{2} \delta_{b}^{2}}=1-\frac{l^{2}}{4}$, where $l$ is an integer, we choose to be equal to one. One can determine the growth rate of the $\mathrm{KH}$ instability in presence of TIM flow in the neighborhood of the stable solution using a Taylor expansion, thus 


$$
C=\frac{\omega}{n}=\left.\frac{\omega}{n}\right|_{n=n_{s}}+\left(n-n_{s}\right)\left(\frac{\partial C}{\partial n}\right)_{n=n_{s}} .
$$

Using the original treatment of Drazin and Howard in Ref. 37 or Shoucri in Ref. 38, we use the following formula to determine $\left.\frac{d C}{d n}\right|_{n=n_{s}}$ (and where PP refers to the principal part):

$$
\begin{aligned}
\left.\frac{d C}{d n}\right|_{n=n_{s}}= & \frac{-\int_{0}^{2 \pi} \varphi_{s}^{2} d x}{P P \int_{0}^{2 \pi} \frac{\omega_{0}^{\prime \prime}(x) \varphi_{s}^{2} d x}{\left(\omega_{0}(x)-\left\langle\bar{\omega}_{d}(\kappa) E\right\rangle_{\kappa, E}\right)^{2}}} \\
& +i \pi \operatorname{sgn} \omega_{0}^{\prime}\left(x_{s}\right)\left(\frac{\varphi_{s}^{2} \omega_{0}^{\prime \prime \prime}\left(x_{s}\right)}{\omega_{0}^{\prime 2}\left(x_{s}\right)}\right) .
\end{aligned}
$$

Thus, the system is expected to be unstable for every toroidal number $n<n_{s}$ with a growth rate given by

$$
\frac{\gamma}{n} \simeq\left(n_{s}-n\right) \frac{n_{s} \rho_{s}^{2}}{4 \pi^{2} \delta_{b}^{2}}
$$

At least, for the set of physical parameters used in the simulation, the numerical value was found in good agreement with the predicted theoretical value.

\section{Interchange-KH coupling regime}

We consider now the following shear flow defined by:

$$
\phi_{0}(\psi)=\frac{3}{2} \omega_{d 0} \psi+\frac{\omega_{d 0} \Delta \psi}{2 \pi} \phi_{\max } \sin 2 \pi \psi
$$

or equivalently

$$
\omega_{0}(\psi)=\frac{3}{2} \omega_{d 0}+\omega_{d 0} \cos (2 \pi \psi) .
$$

Again, we have modified the equilibrium condition to take into account the KH-TIM coupling regime. The initial data (without perturbation) are now chosen to

$$
\begin{aligned}
F_{e q}(\psi)= & e^{-\frac{E}{T_{0}}}\left(1+\left(\frac{5}{2}-\frac{E}{T_{0}}\right) h(\psi)\right) \\
& +e^{-\frac{E}{T_{0}}}\left(\frac{E}{T_{0}}-\frac{3}{2}\right) g(\psi) \triangle \tau \omega_{d 0},
\end{aligned}
$$

where we have introduced the functions $h(\psi)=2 \pi C_{i}$ $\delta_{b}^{2} \phi_{\max } \sin 2 \pi \psi$ and $g(\psi)=\frac{\sin 4 \pi \psi}{8 \pi}+\frac{\psi}{2}$. Using Eq. (12) and the equilibrium (14) leads to the following values of the density and pressure at equilibrium:

$$
\begin{aligned}
& \rho_{0}(\psi)=2 \pi C_{i} \delta_{b}^{2} \phi_{\max } \triangle \tau \omega_{d 0} \quad \text { and } \\
& P_{0}(\psi)=n_{0} T_{0}\left(1+g(\psi) \triangle \tau \omega_{d 0}\right) .
\end{aligned}
$$

From the expression of pressure given in Eq. (15), the calculation of its derivative is straightforward, giving $P_{0}^{\prime}(x)=\frac{\Delta \tau}{2 \pi}$ $\cos x$ and Eq. (1) reduces to

$$
\cos x\left(\varphi_{s}^{\prime \prime}(x)+\left(1-k_{s}^{2}-N_{s}^{2}\right) \varphi_{s}(x)\right)=0,
$$

where $N_{s}^{2}=\frac{3}{2} \frac{\Delta \tau}{C_{i} \delta_{b}^{2} 4 \pi^{2}}$. In subsequent operations, we will ignore the factor $\cos x$ in (16). Finally, we have again for eigenfunction the same solution $\varphi_{s}=\sin \frac{l \pi}{2}$. Thus, the nature of the eigenfunction is not modified. The unstable mode is recovered for $n<n_{s} \simeq \frac{\delta_{b}}{\rho_{s}}\left(3 \pi^{2}-\frac{3}{2} \frac{\Delta \tau \omega_{d 0}}{C_{i} \delta_{b}^{2}}\right)^{\frac{1}{2}}$.

\section{Parametric-type scattering involving ZF and resonant CTIM}

Zero-frequency $\mathrm{ZF}$ is quite non resonant being relatively easy to drive up by the Reynolds tensor in a nonlinear way. While ZFs back-react upon turbulence by shearing, weakening the source of their generation, shear KH type instability can be considered as a damping mechanism for ZFs, which breaks up ZFs. However, this latter mechanism is weakened by the oscillatory nature of ZFs in the resonant regime of CTIMs, which becomes sensible to resonant amplification via parametric scattering. Thus, the problem of $\mathrm{ZF}$ amplification by parametric resonance may have a wide and deep impact on turbulence.

A three-wave coupling mechanism was also observed in simulation: the decay of the interchange-type TIM mode (referred as the pump) $\left(n, \omega_{T}\right)$ into a resonant CTIM mode $\left(n, \omega_{R}\right)$ and a low-frequency oscillating $\mathrm{ZF}(0, \Omega)$. The resonant conditions

$$
n=n+0 \quad \text { and } \quad \omega_{T}=\omega_{R}+\Omega+\delta \omega
$$

are well satisfied. The frequency of the interchange mode is usually considered as close to zero. However, in terms up to $\frac{1}{\omega^{3}}$ are kept in the dispersion relation in the asymptotic expansion, we find a linear frequency close to $\omega_{T} \simeq \frac{5}{2} n \omega_{d 0}$ (see for instance Refs. 39-41 for more details). It was proposed that CTIMs can propagate now in the ion direction, allowing strong resonance with precessing ions. In that case, the linear frequency $\omega_{R}$ is somewhat reduced and is found close to

$$
\omega_{R}=\frac{5}{2} n \omega_{d 0}+\frac{\sqrt{2 \varepsilon} \omega_{* e}}{2(1+\tau)} \simeq \frac{3}{2} n \omega_{d 0},
$$

and now it is possible to amplify a low-frequency ZF mode at a frequency close to $\omega_{T}-\omega_{R} \simeq-\frac{\sqrt{2 \varepsilon} \omega_{* e}}{2(1+\tau)} \simeq \Omega$, where $\omega_{* e}<0$ is the electron diamagnetic frequency.

In (17), we have assumed perfect toroidal number matching (since the simulation box is periodic in $\alpha$ ) and match mode numbers exactly, where $\delta \omega$ contains the mismatch (if any). In the three-oscillator model described in Appendix B, any mismatch in frequency imposed by the finite spatial grid or by nonlinear effects results in some growth reduction (called de-tuning) as the system adjusts itself to achieve exact frequency mismatch and phase locking. The resonance of CTIM is expected to place for a given value of the energy $E_{\text {res }} \sim \frac{\omega_{R}}{n \bar{\omega}_{d}\left(\kappa_{\text {res }}\right)} \sim \frac{3}{2} T_{0}$ and for a given population with a pitch-angle (or trapping parameter) $\kappa_{\text {res }}$, usually different of the set of parameters $\kappa$ and $E$ met in the case of the (fluid) interchange mode. Thus for a given toroidal number $n$, both modes (resonant CTIM and TIM interchange) may coexist. It must be pointed out that only 16 trapped particle populations seem to be sufficient to take into account such the resonant character. 
To end this discussion, it must be pointed out that although such ZF mode has no linear counterpart, it was recently proposed in Ref. 42 that the zonal flow can be also excited by resonant interaction with granulation-type modulation through polarization charge flux. The underlying physics is then based on the phasestrophy concept and its conservative form equivalent to the Charney-Drazin momentum theorem. Taking into account the shear flow, which may alter the resonant condition on $E_{r e s}$ in the dispersion relation, the authors found an estimation of the modulation frequency of $\omega \simeq \frac{\sqrt{2 \varepsilon}\left|\omega_{*}\right|}{1+\tau^{-1}-\sqrt{2 \varepsilon}}$ which indeed is very close to $\omega_{T}-\omega_{R}$ using $\tau=\frac{T_{e}}{T_{i}} \sim 1$ and $\sqrt{2 \varepsilon} \ll 1$.

\section{NUMERICAL SIMULATIONS}

In this section, numerical simulations have been performed in order to elucidate some of the key features of the $\mathrm{CTIM} / \mathrm{KH}$ coupling. We focus on the nonlinear generation of zonal flows by treating KH modes and CTIMs on an equal footing. For this reason, an initial shear flow is defined by Eq. (12), while the initial distribution function given by Eq. (14) is used, with a perturbation given by $\delta f=e^{-E} \delta \phi_{\max }$ $2 \pi C_{i} \delta_{b}^{2} \sin (\pi \psi) \sum_{n=1,5} \cos (n \alpha)$ with $\delta \phi_{\max }=10^{-4}$ to start up the instability.

In summary, our kinetic trapped ion model is given by the set of $N=N_{\kappa} \times N_{E}=16 \times 128$ reduced Vlasov equations coupled together through the quasi-neutrality equation. Each particle bunch defined by the data of the energy $E$ and the trapping parameter (the pitch-angle) $\kappa$ (here with $\kappa<1$ for trapped ions) is self-consistently coupled to the electric potential $J_{0} \phi$, where $J_{0}$ is the gyro-average operator, approximated by the Pade approximation (see Refs. 43, 44 for more details). In simulations, the time is normalized to the inverse precession drift frequency $\omega_{d 0}^{-1}$, the poloidal flux is given in $\Delta \psi$ units (where $\Delta \psi=\frac{r_{0} R_{0} B_{0}}{q_{0}}$ ), the energy $E$ is given in unit $T_{0}$ (thermal ion velocity), and the electric potential is expressed in $\Delta \psi \omega_{d 0}$ units. Here, we have $\omega_{d 0}=\frac{q_{0} T_{0}}{e r_{0} R_{0} B_{0}}$. Simulations were carried out with an initial temperature gradient of $\Delta \tau=0.15$, a value taken above the threshold of the ITG instability. Except the amplitude of the initial shear flow $\phi_{\max }$, all the simulations performed here use the following physical parameters: $C_{e}=0.10, C_{i}=1$, a banana width of $\delta_{b}=0.10$, a (thermal) Larmor radius of $\rho_{s}=0.02$ in normalised units. The time step is $\Delta t \omega_{d 0}$ $=0.001$. Here, we refer to nonlinearly generated streamers and not the (trivial) linear streamer structures (here referred as collisionless TIMs) driven by the ITG instability when only trapped ions are implicated. Two other coherent structures have been observed in simulations: KH modes and zonal flows. In particular, semi-Lagrangian Vlasov simulations have identified two different kinds of ZFs of somewhat different natures, the former coming from KH instability and the latter coming from the resonant coupling with CTIMs.

\section{A. The dominant Kelvin-Helmholtz regime}

It is illuminating to consider the behaviour of the system in detail as $\phi_{\max }$ varies. We choose to start first with a high
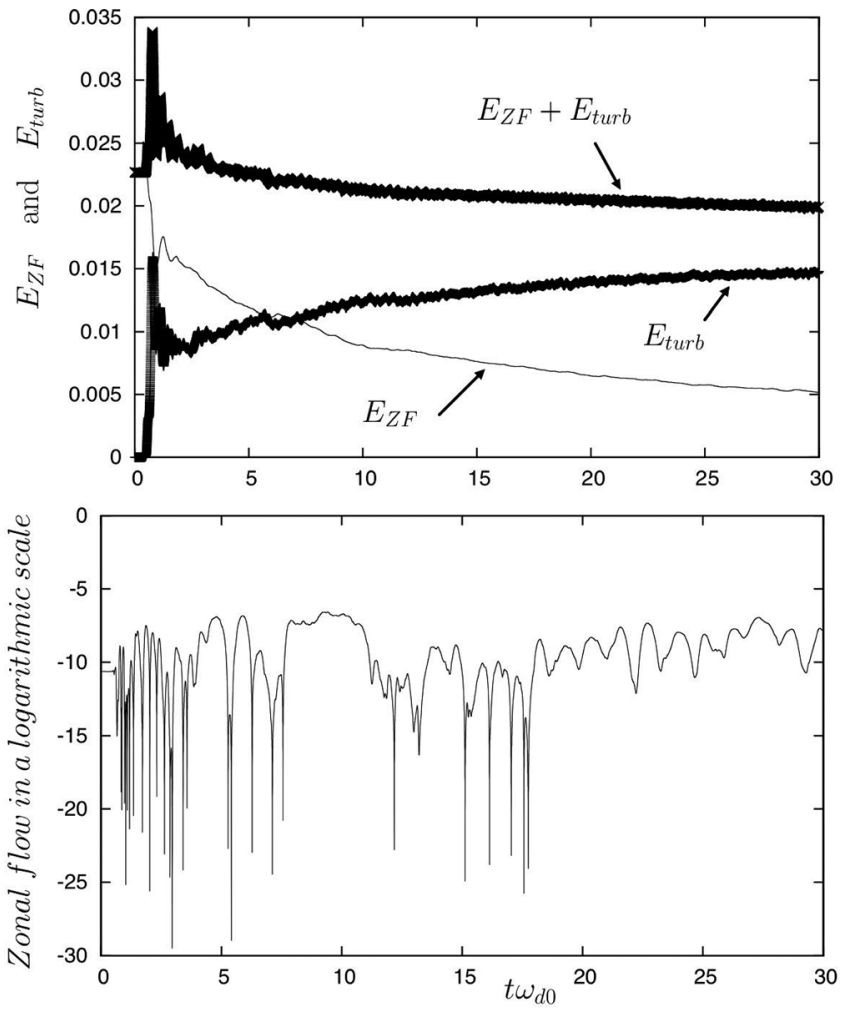

FIG. 1. Time evolution of the zonal flow energy, the turbulent energy, and their mutual sum in the dominant adiabatic KH regime. On bottom panel, the evolution of the zonal flow in a logarithmic scale. The initial shear flow amplitude is $\phi_{\max }=2.5$.

value of the maximum shear flow potential $\phi_{\max }=2.5$. In the limit of a dominant KH regime (shown in Figs. 1-4), zonal flow damping is then efficient (as can be seen on top panel in Fig. 1). We have also plotted the time evolution of the zonal flow $\langle\phi\rangle_{\alpha}$ in Fig. 1 measured at the middle of the box in $\psi$. The curve has been plotted in a logarithmic scale on bottom panel in Fig. 1. The evolution of the turbulent energy was superimposed in thick line on top panel in Fig. 1 plus their mutual sum. As shown in the figure, the zonal flow is a dynamical quantity determined by the generation and the interaction of other KH modes. Details of the dynamics of ZF in the plane $(\psi, t)$ are shown in Fig. 2.

Except a bursty event observed at time $t \omega_{d 0} \simeq 1$, the $\mathrm{ZF}$ energy is transferred to larger-scale $\mathrm{KH}$ vortices in a

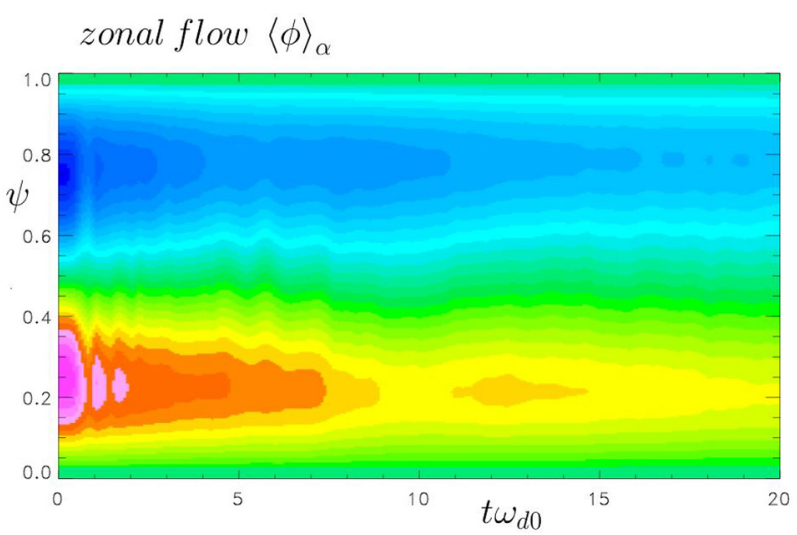

FIG. 2. ZF dynamics in the plane $(\psi, t)$ in the $\mathrm{KH}$ regime of the instability. The initial shear flow amplitude is $\phi_{\max }=2.50$. 
nonlinear and in an adiabatic way. The burst of turbulence is indeed linked to the growth of TIMs followed by streamers, which correspond to the nonlinear oscillating behaviour of the zonal flow observed at that time on the bottom panel in Fig. 1. On the one hand, suppression of turbulence driven by streamers began by considering an initially strong enough imposed ZF which is part of the background. On the other hand, the ZF is also impacted by the formation of large-scale coherent structures referred by Kim and Diamond in Ref. 19 as generalized $K H$ structures ( $G K H$ ), a designation we have kept here to characterize such strong nonlinear coherent structures. Among the four different actors involved in the interaction, only generalized $\mathrm{KH}$ and $\mathrm{ZFs}$ persist in that regime. We examine in Fig. 3 the evolution of the electric potential at three different times. The first plot, at time $t \omega_{d 0}=0.6$ displays at left the excitation of streamers together with the initial perturbation in the central region in phase space. This illustrates a feature of shear flow, which tends to generate large scale structures, clearly observed at time $t \omega_{d 0}=30$. GKH can extract energy from the imposed ZF. One of the key issues in the nonlinear generation of GKH modes is the modification of the flow's theorem. To
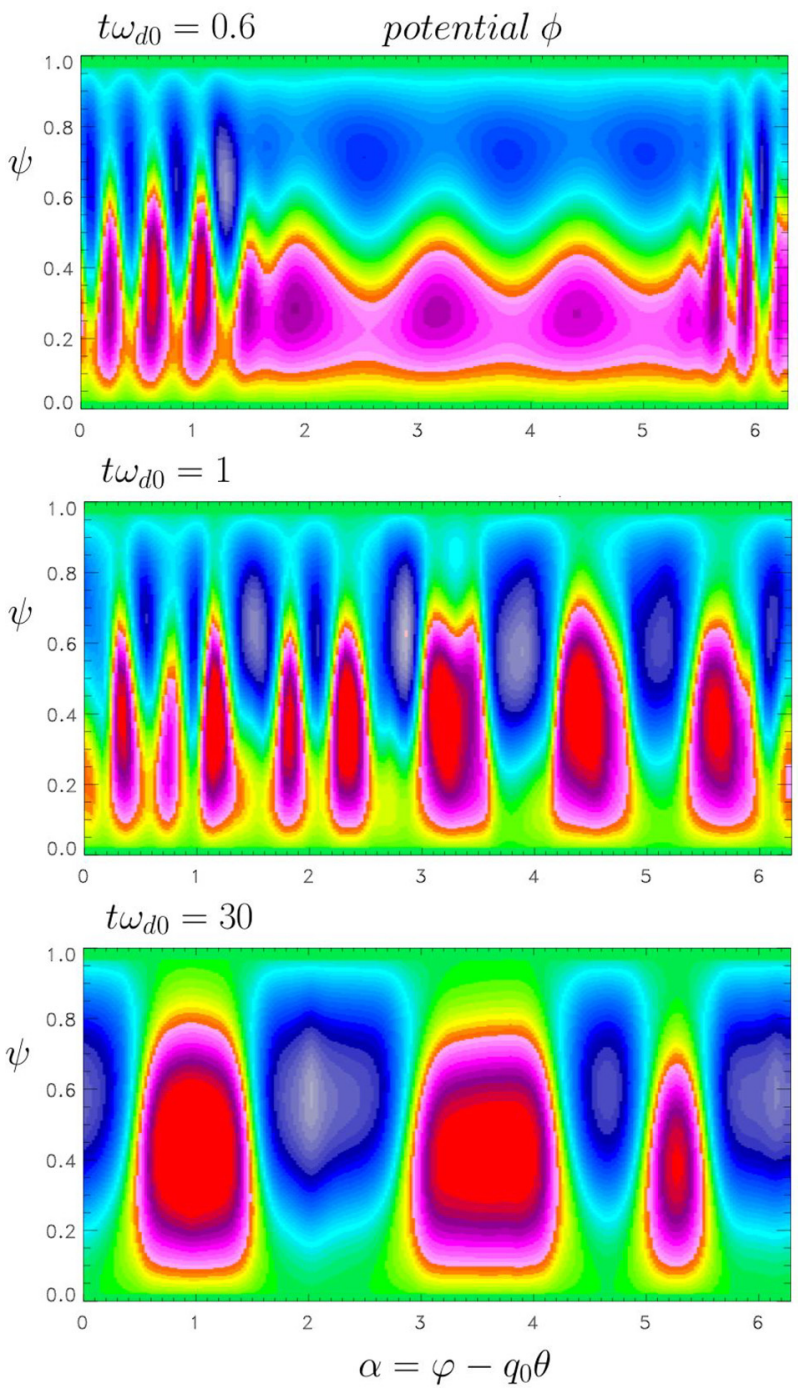

FIG. 3. Plots of the electric potential in the $(\psi, \alpha)$ plane at three different times in the $\mathrm{KH}$ regime for $\phi_{\max }=2.5$. study these issues in detail, we have also displayed the $\frac{E \times B}{B^{2}}$ drift velocity in Fig. 4 at three different times. The top panel shows clearly the topology corresponding to the initial shear velocity. At time $t \omega_{d 0}=4.40$, such topology has changed in a global way with strong flow now in the $\psi$ direction.

Suppression of turbulence and transport by flow shear occurs in the first step of evolution, i.e., for a time $t \omega_{d 0} \leq 4$. Assuming new type of turbulence whose driving source is now unaffected by the background flow shear (such a source is here an instability associated with the shear flow i.e., the $\mathrm{KH}$ instability), the slow growth of the turbulence energy implies a decrease in the ZF energy. This leads to the asymptotic decay of the ZF energy until a new balance is established with $E_{Z F}+E_{\text {turb }}=$ const. Thus, a $2 \mathrm{D}$ vortex structure is nonlinearly formed, stabilized by a modification of the background shear flow (see bottom panel in Fig. 3). More generally, the curvature of the ZF tends to stabilise flowshear driven instabilities. The problem is somewhat similar to that met in the Earth's stratosphere. Large scale shear flows in the stratosphere, such as the equatorial jet (see Ref. 45 ), are stable most of the time. Occasionally, the flow becomes unstable due to an episodic disturbance called a
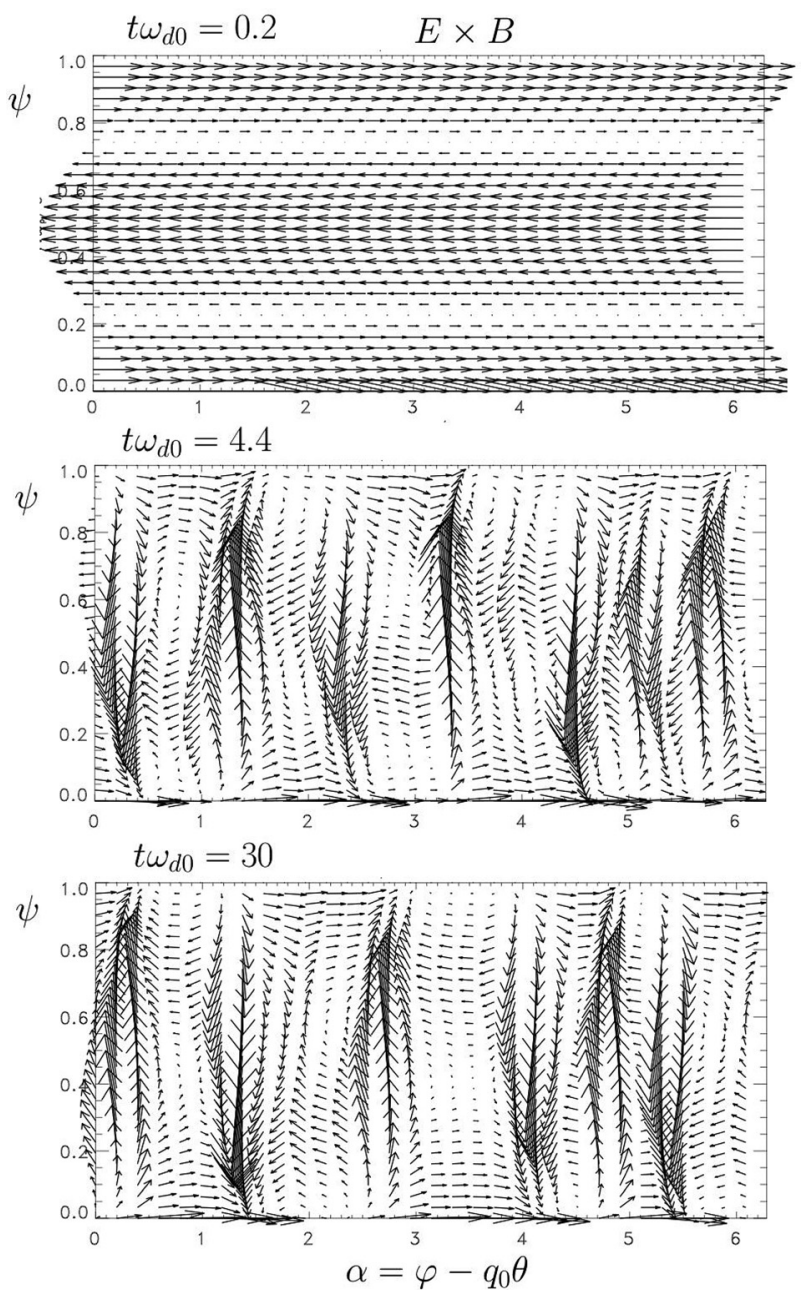

FIG. 4. Modification of the topology of the $E \times B$ drift velocity in the "phase" space plane. The standard KH mode on top panel, while the bottom panel illustrates a "generalized" KH mode. 
wave-breaking event, ${ }^{46}$ which redistributes the flow curvature and stability is re-established.

\section{B. Transition driven by TIM/KH coupling}

We have so far assumed that GKH modes and ZKs play a major role in the TIM/KH coupling. This is of course an oversimplified picture and there are other coherent structures that can be potentially important. As noted before, the counterpart non zero frequency ZFs, CTIMs, and streamers must be taken into account.

The nonlinear generation of ZF by the Reynolds stress is particularly noticeable in situations relevant to ITG instabilities. The above example of Section III A demonstrates that strong KH flow shear stabilizes collective TIM instabilities. However, there is no universal criterion for stabilization, and weaker flow shears can be destabilizing. The feedback mechanisms observed in Vlasov simulations are nonlinear, also indicating that the effect of flow shear on turbulent fluctuations likely involves some combinations of many actors as streamers, GKH modes, CTIMs, and time-varying ZFs.

We choose now to decrease the flow shear amplitude $\phi_{\max }$ to 0.25 , keeping others physical parameters identical. This subsection describes aspects of the phenomenology of transitions in order to illustrate the rich nonlinear dynamics that underlies the TIM/KH coupling. Moreover, the turbulent energy shows three different steps during its evolution: a first step for $t \omega_{d 0} \leq 10$ followed by a second (weak) peak at $t \omega_{d 0} \simeq 10$. Then we observe a significant reduction of turbulence in the temporal range $[10,15] \omega_{d 0}^{-1}$. Here, the Reynolds tensor is however not sufficient to drive a steady flow, whose shear suppresses completely turbulence. Meanwhile, a remarkable intermittent behaviour in turbulent energy appears, accompanied by the occurring of strongly nonlinear streamers which now dominate the turbulence dynamics.

In the ITG instability, the free energy for TIMs is provided by the ion temperature gradient. At the instability threshold, where the temperature gradient scale length just exceeds a critical value $\left(\triangle \tau_{\text {threshold }} \sim C_{e}\right)$, the gradient free energy is accessed through a resonance of the mode with the banana orbits under the drifts produced by the gradient and the curvature of the magnetic field. Now the imposed flow shear is destabilizing at weak levels. A simple illustration of this process can be easily observed from the time evolution of both $\mathrm{ZF}$ and turbulent energies shown on top panel in Fig. 5.

The corresponding bottom panel in Fig. 5 exhibits the time behaviour of the ZF on a logarithmic scale. In addition to these diagnostics, we have also displayed the behaviour of the ZF in the poloidal flux $(\psi)$-time $(t)$ plane in Fig. 6. In Figs. 5 and 6 , a first bifurcation of the plasma state is evident at time $t \omega_{d 0} \simeq 10$, manifested as an abrupt change of the $\mathrm{ZF}$ dynamics. Note that at that time, the ZF energy increases and that the ZF is typically generated by the Reynolds stress and is characterized by a zero-frequency behaviour on bottom panel in Fig. 5 (which seems indicate that the Reynolds stress is the dominant source).

An overview of the $(\psi, \alpha)$ space behaviour of the electric potential, we will examine in detail, is given in Figs. 7-9
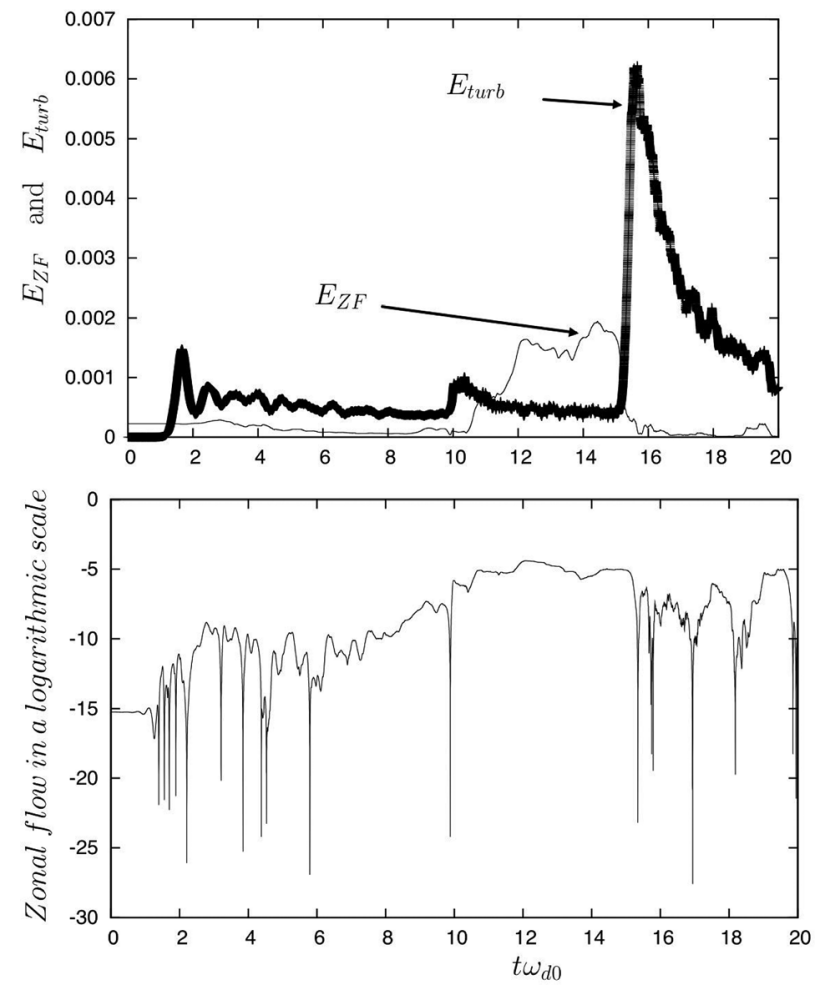

FIG. 5. On top panel, the time evolution of the zonal flow energy in solid line and the turbulent energy (in thick line) for the interchange CTIM regime. We observe clearly a turbulent burst for $t \omega_{d 0} \simeq 15$. On bottom panel, the time evolution of $\mathrm{ZF}\langle\phi\rangle_{\alpha}(\psi \sim 0.5, t)$ in a logarithmic scale showing the mixing of both $\langle\phi\rangle_{Z F, K H}$ and $\langle\phi\rangle_{Z F, C T I M}$ contributions. The amplitude is here $\phi_{\max }=0.25$.

for the three different steps mentioned above. Fig. 7 exhibits the occurring and the growth of nonlinear GKH modes as the result of the shear flow instability. In Fig. 7, the top panel shows the initial perturbation mode on the five first modes, followed on the middle panel by the excitation of the GKH modes at the peak of turbulence at $t \omega_{d 0} \simeq 1.8$, followed on the bottom panel by the saturated state, here dominated by the GKH mode at time $t \omega_{d 0} \simeq 9.8$.

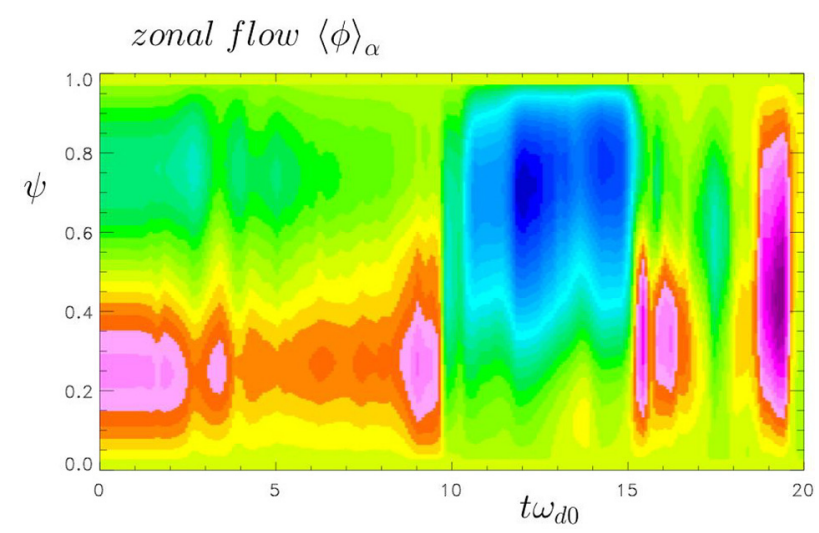

FIG. 6. Poloidal flux-time representation of the zonal flow $\langle\phi\rangle_{Z F}=\langle\phi\rangle_{Z F, K H}$ $+\langle\phi\rangle_{Z F, \text { CTIM }}$ for the interchange-type regime of the instability. Note the change in the time interval $[10,15] \omega_{d 0}^{-1}$ just before the turbulent burst located at $t \omega_{d 0}=15$. The amplitude parameter is taken to $\phi_{\max }=0.25$. 


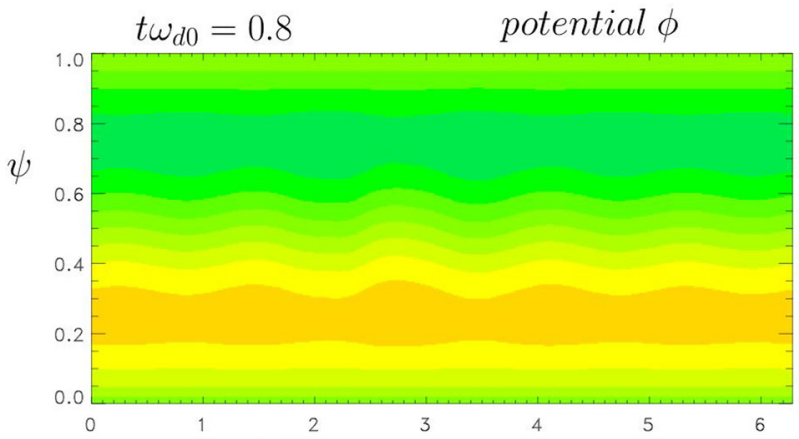

$t \omega_{d 0}=1.8$

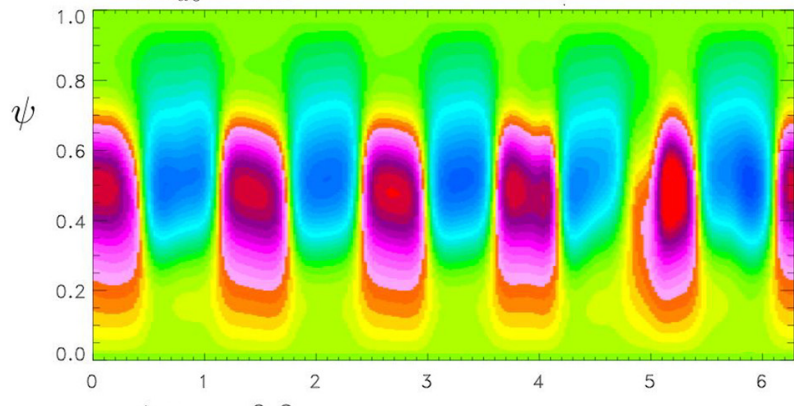

$$
t \omega_{d 0}=9.8
$$

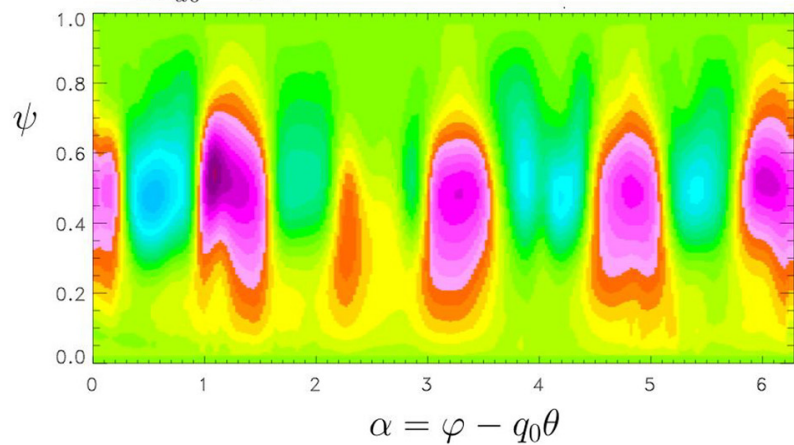

FIG. 7. Electric potential representation at three different times during the first phase of the instability, showing the growth of $\mathrm{KH}$ modes driven by the initial shear flow. The numerical simulation was performed with $\phi_{\max }=0.25$.

A second peak emerges at time $t \omega_{d 0} \simeq 10$ as the result of the growth of TIMs (top panel in Fig. 8), followed by a burst at time $t \omega_{d 0} \sim 15$ due now to nonlinear streamers. In Paper I, we have shown that the zonal flow is typically generated self-consistently by the combined action of the Reynolds tensor and polarization effects (through coupling with pressure). Although the effect of shear flow on TIM will be studied in detail in Section IV C, we can observe that the shear flow is too high and the TIM turbulence is apparently quenched. In Fig. 8, we see clearly that the main actor is here the zero-frequency ZF. This mode which appears as a quasi-coherent feature in the fluctuating electric potential in Fig. 8 was observed to increase in amplitude in top panel in Fig. 5. However, it may be understood that the stabilizing effect of ZF is too weak compared with the strong nonlinear dynamics of streamers, so that it is submerged by strong growth of nonlinear streamers. As the ZF energy is reduced, the corresponding level of turbulent streamer (ITG) fluctuations is high, as can be seen on top panel in Fig. 9 at $t \omega_{d 0} \simeq 15.4$, and the self-generated zonal flow decreases much. It is characterized by the strong intermittent (fast)
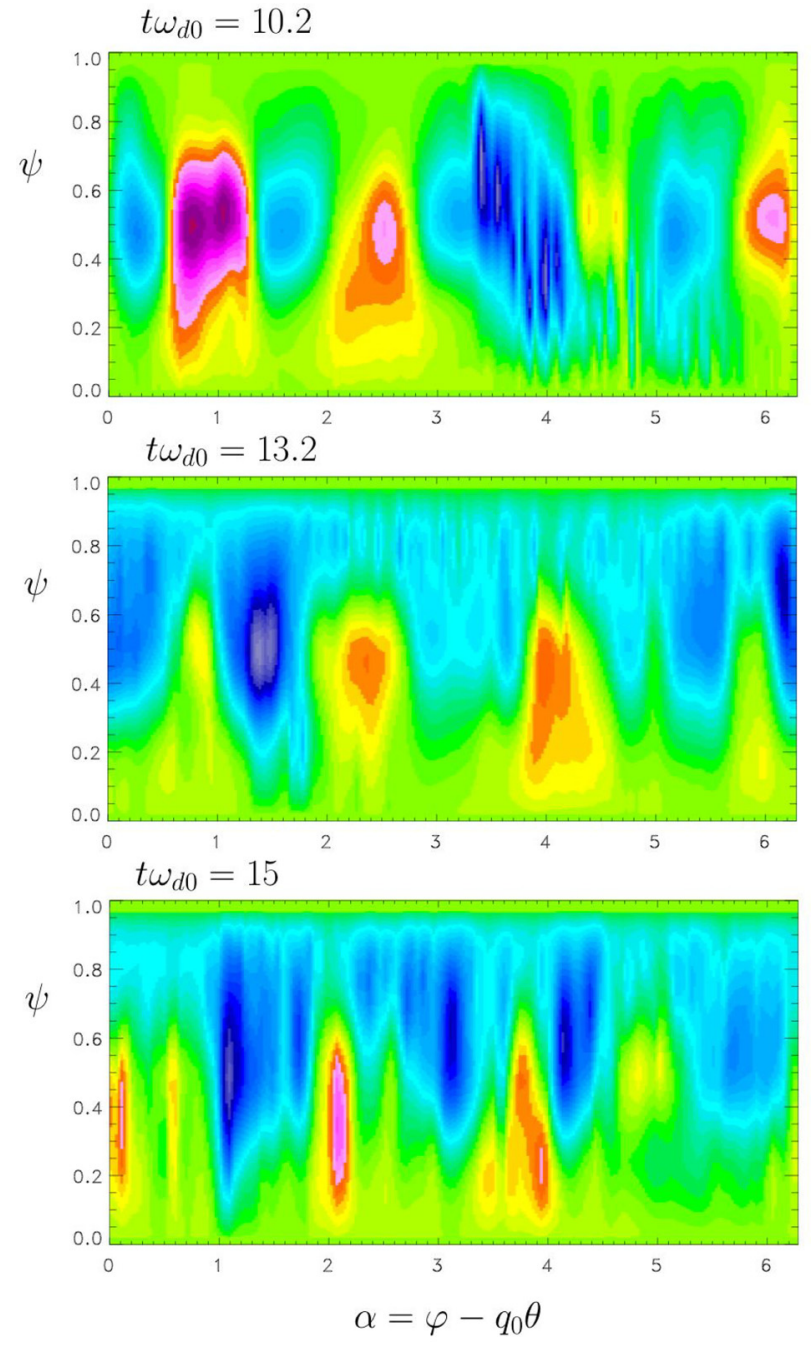

FIG. 8. Continuing the time evolution of the electric potential of Fig. 7, but now when $\mathrm{ZF}\langle\phi\rangle_{Z F, K H}$ is growing and dominates showing strong plasma turbulence reduction.

growth of the turbulent energy. It is clear that the ITGdriven streamer dynamics still dominates in the strong turbulent regime. Finally, the asymptotic state exhibits the emergence of both small-scale fluctuations and large-scale potential structures.

\section{Onset of oscillating ZF and associated dynamics}

We focus now on transition towards the occurring of resonant CTIM, which is induced by the precessional resonance of trapped ions. Thus, wave-particle resonance can lead to the amplification of the mode $\langle\phi\rangle_{\text {ZF,CTIM. In the }}$ Taylor-Goldstein model, the eigenmode equation is like the $\mathrm{KH}$ eigenmode equation with the addition of the curvature drive introduced by $P_{0}^{\prime}(\psi)$. Thus, the advective pressure response in the denominator of the curvature drive complicates the eigenmode structure; hence, a simple characterization of the effect of flow shear is more difficult. However, we expect that there is a regime where the flow shear is too weak to stabilize the interchange instability. We choose here to decrease the initial flow shear amplitude to $\phi_{\max }=0.025$ to enter inside such a regime where the resonance takes 

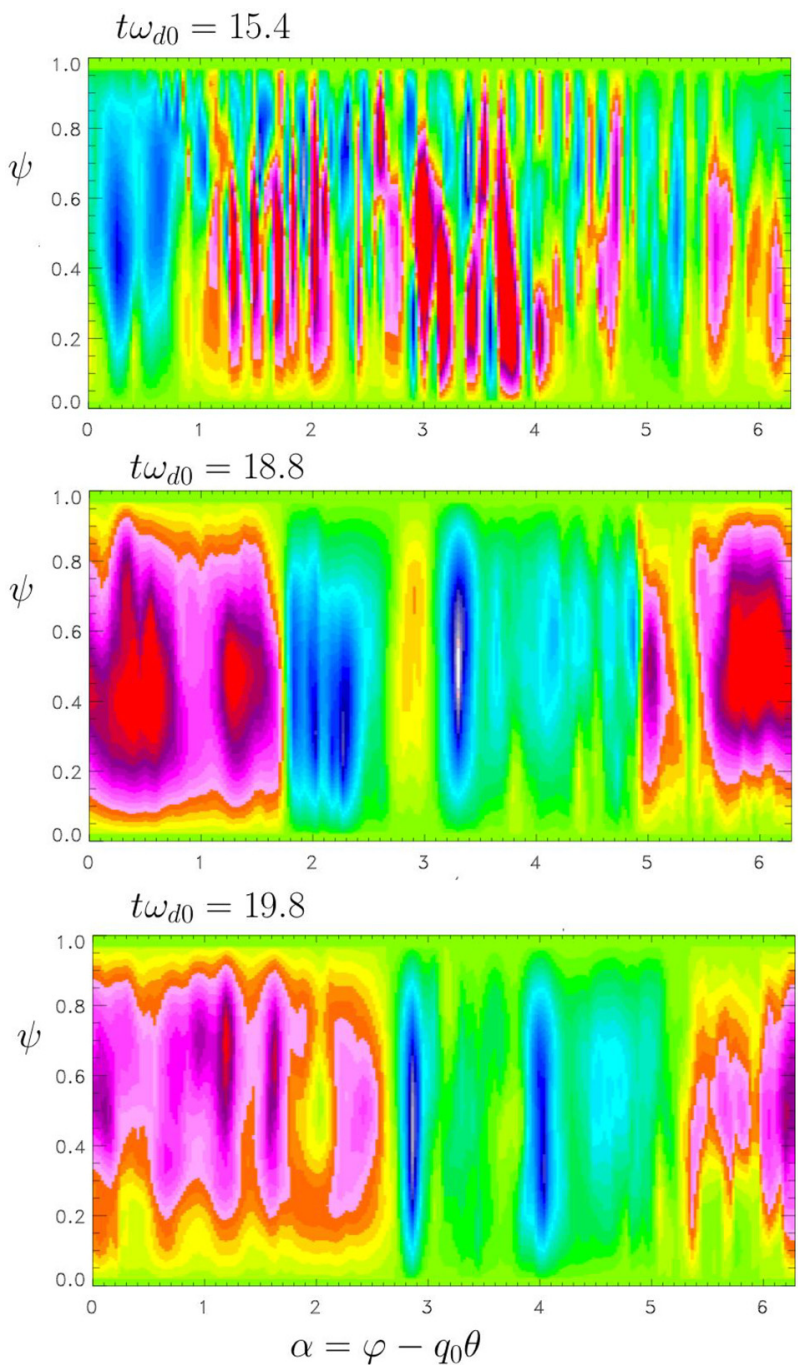

FIG. 9. Continuing the evolution of the electric potential of Figs. 7 and 8 in the regime of the turbulent burst emergence. We see now clearly the formation of strong nonlinear streamers on top panel. A large-scale structure is formed when the turbulent energy decreases.

place. Fig. 10 shows such a temporal behaviour both the time evolution of the ZF and turbulent energy contributions (on top panel) and the mode $n=0$ (the zonal flow) in a logarithmic scale on bottom panel.

An intermittent dynamics has been observed in the behaviour of the turbulent energy characterized by a burst at time $t \omega_{d 0} \simeq 17$. Note that the corresponding time behaviour of ZF exhibits the low-frequency fluctuations, which was not observed previously when the Reynolds stress was the dominant driver. It is interesting to consider the behaviour of the mean potential $\langle\phi\rangle_{\alpha}(\psi, t)$ of the zonal flow in the plane $(\psi, t)$. Fig. 11 illustrates the details of $\langle\phi\rangle_{\alpha}(\psi, t)$, showing the lowfrequency oscillating behaviour of the mode. As the energy of the zonal flow increases slowly, such oscillations are dominant. The abrupt growth in the turbulent energy in Fig. 10 (top panel), observed at time $t \omega_{d 0} \simeq 1.8$, results in the modification of the topology of the drift velocity $\boldsymbol{v}_{E}=\frac{E \times \boldsymbol{B}}{B^{2}}$, where the plasma bifurcates from the initial state dominated by the shear flow (i.e., the KH instability) to another state where the vortex structures is strongly modified.
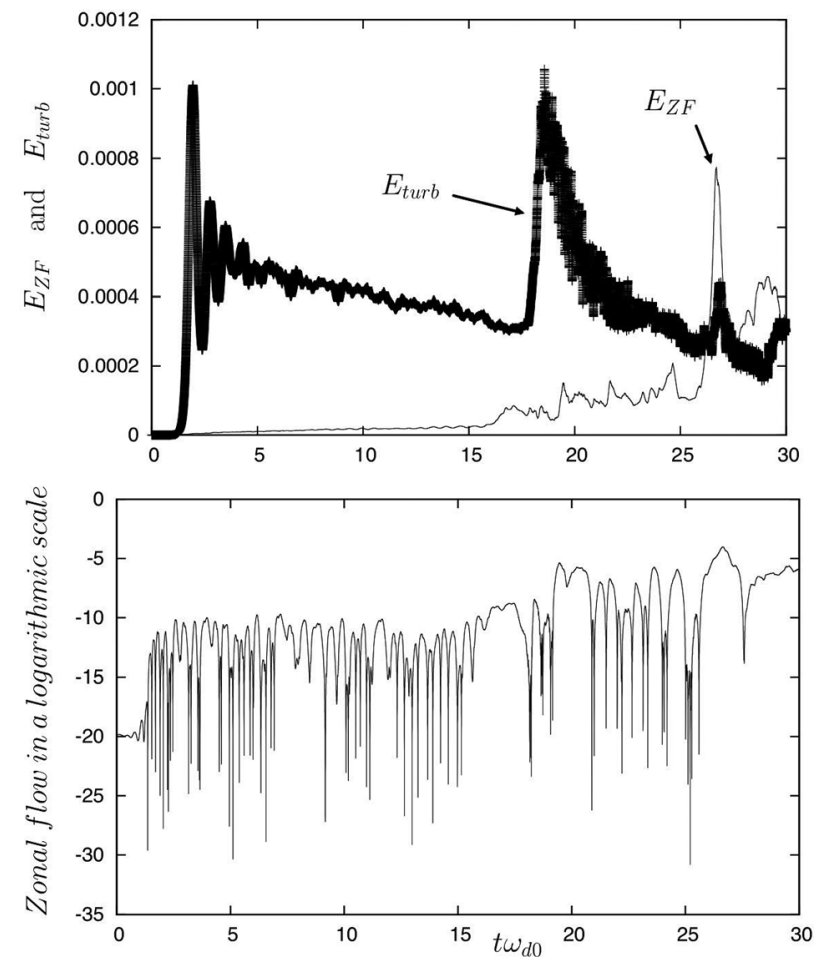

FIG. 10. Similar diagnostics as in Fig. 5 but now in the resonant regime of CTIM, obtained by decreasing the amplitude of the shear flow to $\phi_{\max }=0.025$. We observe the turbulent burst at time $t \omega_{d 0} \simeq 18$ followed by a sharp peak of both zonal plus turbulent energies at $t \omega_{d 0} \simeq 27$ on top panel as the result of a three-wave parametric instability involving CTIMs.

In Fig. 10, on top panel, the third observed sharp peak, observed at time $t \omega_{d 0} \sim 27$, on the $\mathrm{ZF}$ is associated with enhanced turbulence, which seems to indicate that the nature of the process is now different. Such a process results from the parametric decay of an (interchange) CTIM mode into a ZF and a (scattered) resonant CTIM mode. In Fig. 12, we have represented the spectrum in frequency on top of the electric potential, calculated in the time interval $[0,20] \omega_{d 0}^{-1}$ (i.e., before the scattering takes place), while the bottom panel curve corresponds to the global spectrum for the time interval $[0,30] \omega_{d 0}^{-1}$. Note that the accuracy in spectrum

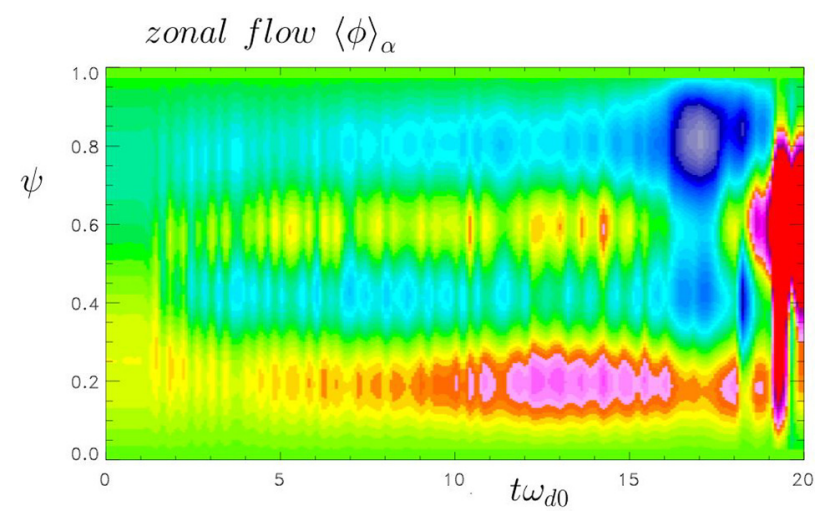

FIG. 11. Details of the dynamics of ZF in the space $\psi, t$, showing the oscillatory behaviour of the zonal flow. The simulation was carried out by decreasing $\phi_{\max }$ to 0.025 . 


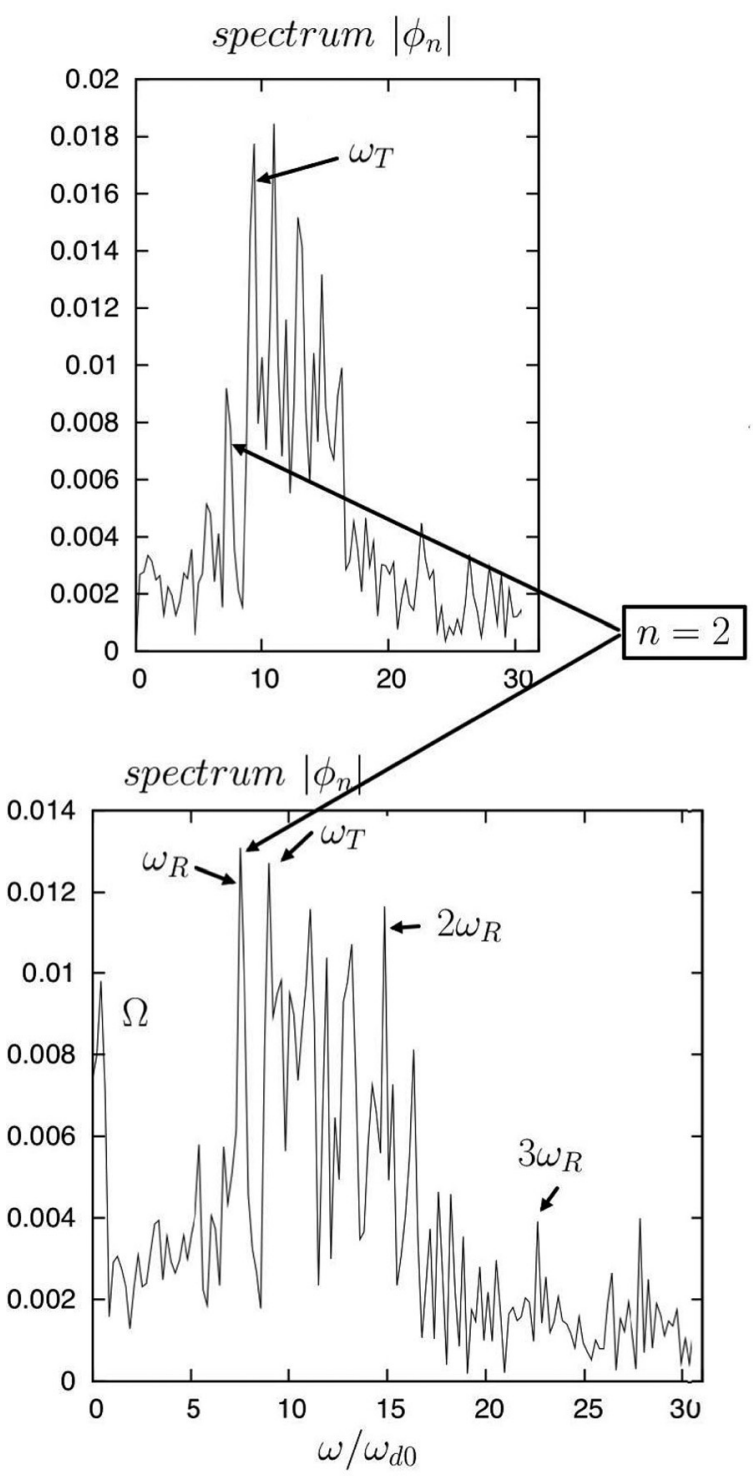

FIG. 12. Spectra in frequency of the electric potential calculated, respectively, in the time interval $[0,20] \omega_{d 0}^{-1}$ on top (before the resonance arises) and in the time interval $[0,30] \omega_{d 0}^{-1}$ on bottom. We see clearly that the growth of $\mathrm{ZF}$ is connected to the growth of the resonant mode $\omega_{R} \simeq 7.60 \omega_{d 0}$. Note also the emergence of the second $2 \omega_{R}$ and third $3 \omega_{R}$ harmonics. During the growth note, the decrease in the interchange mode $\omega_{T}$ (considered as the pump) and the growth of the $\mathrm{ZF}$ at $\Omega \simeq 0.90 \omega_{d 0}$. Here, we have $\phi_{\max }=0.025$.

calculation is close to $\frac{2 \pi}{T_{\max }} \sim 0.2 \omega_{d 0}$. The top panel exhibits the different interchange and GKH modes frequencies. In the considered interval, the resonant peak, corresponding to the toroidal number $n=2$, remains at weak level. If we focus on the spectrum for frequencies weaker than $10 \omega_{d 0}$, we see clearly that when the parametric scattering takes place, two new frequencies are now growing in the frequency range and are located on the frequencies $\omega_{R} \simeq 7.60 \omega_{d 0}$ (for the resonant CTIM mode) and the zonal flow component $\omega_{Z F, \text { CTIM }} \simeq 0.90 \omega_{d 0}$, while the interchange mode $\omega_{T} \simeq 8.70 \omega_{d 0}$ (the pump) decreases in intensity in this time interval associated with the occurring of the parametric instability.

From the linear analysis of the dispersion relation, we can obtain an estimation of the interval in frequency of the interchange mode. The observed frequencies corresponds to a value of the toroidal number of $n=2$. The frequencies, observed numerically have been found in good agreement with the theoretical frequencies given by $\omega_{T} \simeq \frac{5}{2} n \omega_{d 0}\left\langle\bar{\omega}_{d}(\kappa ; s)\right\rangle_{\kappa}$, whose mean value is given by $\omega_{T} \simeq 10,5 \omega_{d 0}$, after integrating over the pitch angle parameter. Note that the frequency range of the interchange mode is $\omega_{T} \in[5.0,14.0] \omega_{d 0}$ for the mode $n=2$ for the different values of the pitch-angle $\kappa<1$ (in simulation we have cut $\kappa$ to a limit value of $\kappa_{\max }=0.95$ and $\bar{\omega}_{d} \in[1,2.8] \omega_{d 0}$ for a value of the magnetic shear of $s=2$ ). Using Eq. (18), an estimation of the mean resonant frequency of CTIM is close to $\omega_{R} \simeq 8.15 \omega_{d 0}$. Thus, we estimate that the mean value of the ZF frequency given by $\Omega_{t h}=\omega_{T}-\omega_{R} \simeq 1.10 \omega_{d 0}$ found in good estimation with the numerical estimation of $\Omega \simeq 0.90 \omega_{d 0}$. The last point concerns the observation in the spectrum on bottom in Fig. 12 of the two first harmonics of the resonant mode obtained for $2 \omega_{R} \simeq 15.2$ and $3 \omega_{R} \simeq 22.8 \omega_{d 0}$.

This mechanism is analyzed in more detail through the diagnostics of Figs. 13 and 14. Fig. 13 shows the evolution of the shear flow during the first step of turbulent growth. Here, we have found that the resonance introduces an

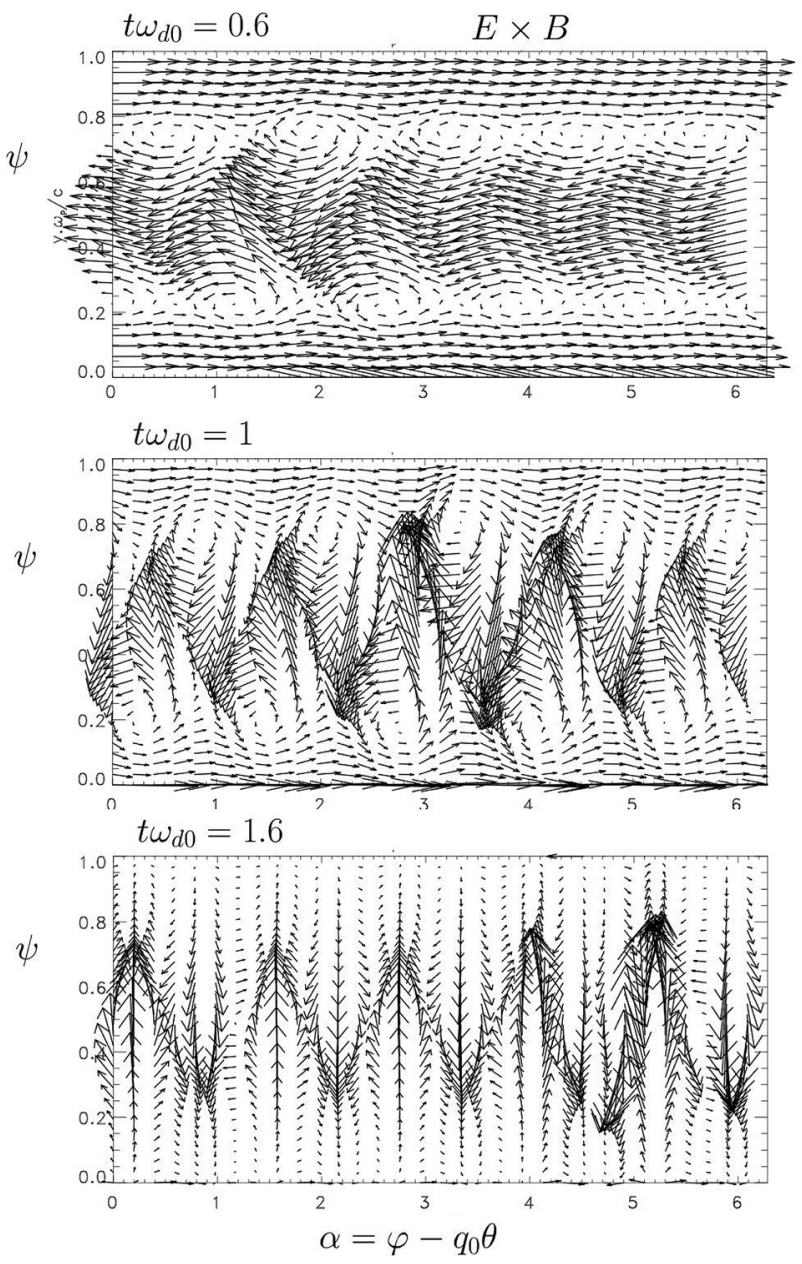

FIG. 13. Behaviour of the drift $E \times B$ velocity at three different times. The first peak in turbulence corresponds to the formation of an interchange CTIM mode at time $t \omega_{d 0} \simeq 1.80$. The transition is observed from the initial shear flow driven $\mathrm{KH}$ (top panel) towards the interchange mode on bottom panel. The simulation was performed with $\phi_{\max }=0.025$. 

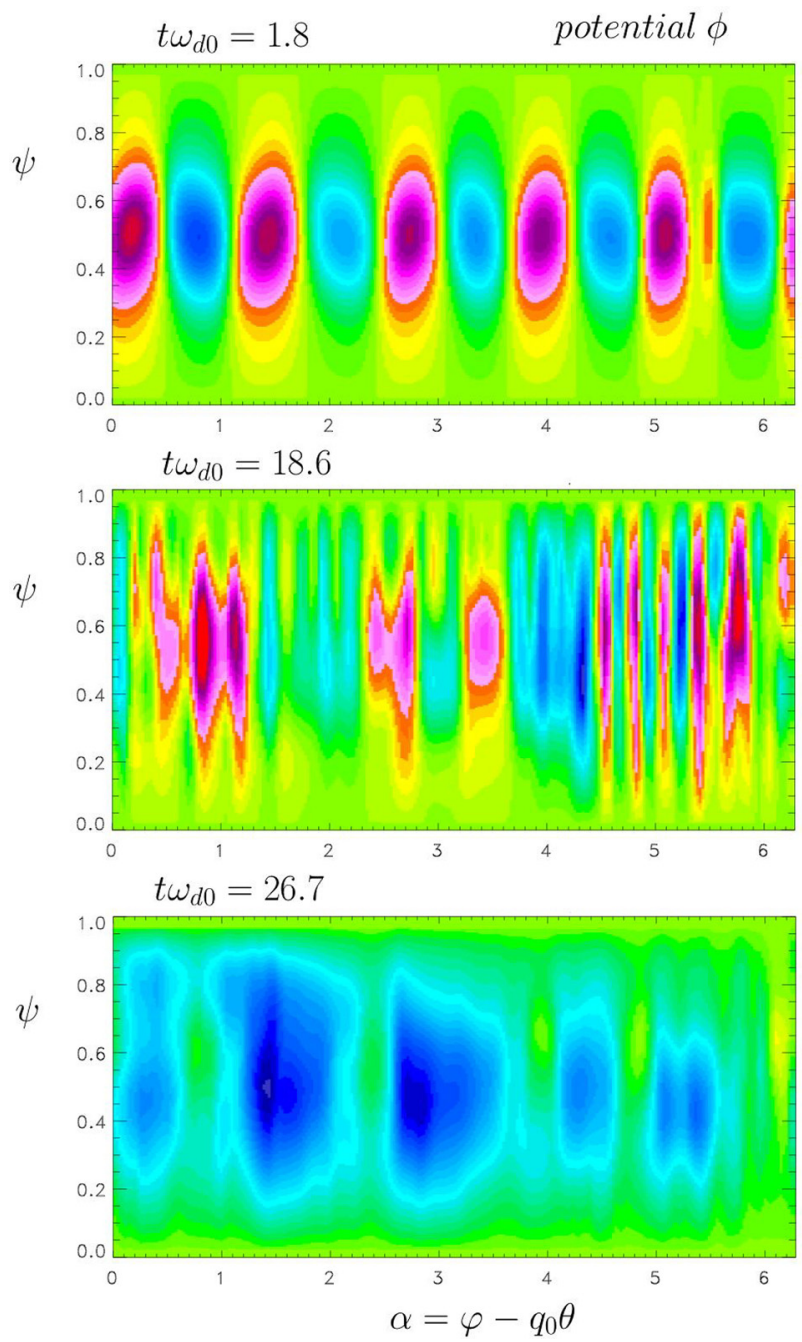

FIG. 14. Corresponding plots of the electric potential at three different times during plasma evolution: on top, the occurring of the interchange mode on $n=5$ at time $t \omega_{d 0}=1.8$ corresponding to the first sharp peak in Fig. 10, followed by the emergence of streamers (on middle) during the burst of turbulence located at $t \omega_{d 0} \simeq 18.6$ towards the occurring of the CTIM-driven ZF emergence at time $t \omega_{d 0} \simeq 26.7$ (on bottom) when the resonance takes place. Note that turbulence is completely quenched by (time varying) ZF at that time.

alternative state dominated by oscillating ZF near the critical temperature gradient. The first peak of growth reaches a maximum at time $t \omega_{d 0} \simeq 1.8$, which corresponds to the formation of an interchange mode, clearly observed in Fig. 13 on bottom panel. We see clearly that the initial vortices induced by the initial shear flow (through the $\mathrm{KH}$ instability) are now disappeared and leaded to plasma flow in a perpendicular direction. In Fig. 14, we have plotted three different snapshots of the electric potential. The top panel corresponds to the formation of the interchange - CTIM structures obtained on the toroidal mode $n=5$. The intermittence is characterized by occurring of a burst at time $t \omega_{d 0} \simeq 17$ as the result of the emergence of the resonant CTIM on the toroidal number $n=2$. Note that the streamers are also occurring but on higher mode $n \sim 23$. Accumulating energy through the second burst, induced by the three-wave process, the zonal flow energy reaches the value enough to quench turbulence at time $t \omega_{d 0} \simeq 26.7$. The corresponding snapshot for the potential in Fig. 14 shows clearly that ZF is now the dominant mode (on bottom panel).

Such a feature is considered to originate from the resonance mechanism driven by the beating of both CTIMs (interchange plus kinetic resonant part) made on the same toroidal number $n=2$. Here, we observe that the abrupt growth of both $E_{Z F}$ and $E_{\text {turb }}$ is occurring simultaneously at time $t \omega_{d 0} \simeq 26.7$. In contrast to the two previous first phases of the instability, the three-wave process exhibits a very sharp peak characteristic of the resonance.

\section{Summary of numerical results}

These various results are summarized in Table II. The level of interaction is controlled by the data of the initial amplitude mean shear flow $\phi_{\max }$. With decreasing the initial mean shear flow, the KH instability is reduced. Evidence is presented that the low-frequency GAM-type ZF might play a major role in the $\mathrm{LH}$ transition. There is also a qualitative change in the interchange-type turbulence behaviour which drops and becomes more intermittent with short semirandom pulses. The $\mathrm{KH}$ actor allows us to couple zerofrequency $\mathrm{ZF}$ of hydrodynamical nature with this new branch of (nonlinear) low-frequency GAM-type mode induced by magnetic trapping. These results are consistent with the recent experimental observation of low-frequency ZFs in EAST tokamak.

TABLE II. Table of results obtained by direct numerical simulation using the trapped ion model, for successive regimes determined by the initial shear amplitude parameter.

\begin{tabular}{|c|c|c|c|}
\hline Dominant interaction regime & Key issues for energy transfer & Coherent structures & Examples \\
\hline KH regime & ZF energy decreases & Generalized KH modes & Fig. 3 bottom \\
\hline Level of initial shear flow: 2.5 & Turbulent energy increases & $\begin{array}{c}\text { Low-frequency ZFs } \\
\text { L mode }\end{array}$ & Fig. 2 \\
\hline Intermediate CTIM/KH regime & No three-wave interaction & Generalized KH modes & \\
\hline Level of initial shear flow: 0.25 & $\begin{array}{l}\text { ZF energy decreases } \\
\text { Turbulent energy increases } \\
\text { Bursts in turbulent energy }\end{array}$ & $\begin{array}{c}\text { +nonlinear streamers } \\
\text { both fluid plus kinetic ZFs } \\
\text { L mode }\end{array}$ & $\begin{array}{l}\text { Fig. } 9 \text { top } \\
\text { Fig. } 6\end{array}$ \\
\hline $\begin{array}{l}\text { Magnetic trapping induced } \\
\text { Low-frequency ZFs } \\
\text { Level of initial shear flow: } 0.025\end{array}$ & $\begin{array}{l}\text { Resonance takes place } \\
\text { Three-wave interaction } \\
\text { ZF energy increases }\end{array}$ & $\begin{array}{l}\text { Turbulence is quenched } \\
\qquad \mathrm{H} \text { mode }\end{array}$ & Fig. 14 bottom \\
\hline
\end{tabular}




\section{CONCLUSION}

In conclusion, a new trapped-particle-induced geodesic acoustic mode is shown to occur using a gyrokinetic reduced model based on a double gyro-average on both cyclotron and bounce motions. The mode is driven by magnetic trapping effects and linked to the resonant character of CTIMs. These results constitute a necessary theoretical basis to understand the low-frequency GAM-type ZF excitation in a global gyrokinetic code (including circulating ions). Therefore, these papers must be understood as a first step in the route towards the possible interaction between low-frequency ZFs, GAMs, and turbulence.

Applications of the model to CTEM are in principle possible. The effects of coupling between shear flow-driven $\mathrm{KH}$ and CTIMs are modeled in terms of eigenfunctions leading to the Taylor-Goldstein equation, a technique already used to describe the Holmboe instability in stratified shear flow in the Earth's atmosphere.

Within the framework of a simplified picture of turbulence involving ITG instabilities, many actors are implicated as collisionless TIMs, streamers, nonlinear GKH modes, and of course zonal flows, the latter possessing different natures having zero-frequency or/and low-frequency modes close to $\omega_{d 0}$. Because TIM possesses an ion diamagnetic branch, the coupling mechanisms take the form of both non resonant (interchange-type) or resonant interactions. We have emphasized on a triad which plays a leading role in the developing turbulent stage which can enhance the growth of the (resonant) zonal flow, and thus compete with the $\mathrm{KH}$ damping mechanism.

Finally, systematic studies on coupled dynamics among granulations, trapped electron modes (which in principle can excite zonal flow at higher frequencies), and shear flows should be investigated in detail using such reduced analytical model.

\section{ACKNOWLEDGMENTS}

The authors are indebted to the IDRIS computational center, Orsay, France, for computer time allocation on their computers. This work was granted access to the HPC resources (Grant No. 2014-2015- 054028) made by GENCI (Grand Equipement National de Calcul Intensif). This work was carried out within the framework of the European Fusion Development Agreement and the French Research Federation for Fusion studies (FR FCM). It is supported by the European Communities under the Contract No. CfP WP14-ENR-01 of Associations between EURATOM and CEA.

\section{APPENDIX A: THE TAYLOR-GOLDSTEIN EQUATION}

We begin with the fluid version of the trapped ion model using the different moments of the distribution function. By introducing the quantities $\rho=n_{i}-n_{0}$, where $n_{i}$ is the ion density, the ion pressure and the second moment $M_{2}$, we have

$$
\frac{\partial \rho}{\partial t}+[\phi, \rho]+\frac{3}{2} \omega_{d 0} \frac{\partial P}{\partial \alpha}=0
$$

$$
\begin{gathered}
\frac{3}{2} \frac{\partial P}{\partial t}+\frac{3}{2}[\phi, P]+\omega_{d 0} \frac{\partial M_{2}}{\partial \alpha}=0, \\
C_{e}\left(\phi-\langle\phi\rangle_{\alpha}\right)-C_{i} \rho_{s}^{2} \frac{\partial^{2} \phi}{\partial \alpha^{2}}-C_{i} \delta_{b}^{2} \frac{\partial^{2} \phi}{\partial \psi^{2}}=0,
\end{gathered}
$$

where we have assumed, for simplification that $J_{0} \rightarrow 1$ and $\bar{\omega}_{d}(\kappa) \simeq 1$. Here [,] denotes the Poisson bracket defined by $[\phi, n]=\frac{\partial \phi}{\partial \psi} \frac{\partial n}{\partial \alpha}-\frac{\partial \phi}{\partial \alpha} \frac{\partial n}{\partial \psi}$ and $M_{2}=\frac{2}{\sqrt{\pi}} \int_{0}^{\infty} d E \sqrt{E} E^{2} f$. The procedure consists in linearizing Eqs. (A1)-(A3) around the steady-state solution $\rho_{0}(\psi), \phi_{0}(\psi)$, and $P_{0}(\psi)$ assuming a time variation in $e^{-i \omega t}$ and phase perturbation of the form $e^{i n \alpha}$, i.e.,

$$
\left\{\begin{array}{l}
\rho=\rho_{0}(\psi)+\sum_{n} \delta \rho_{n}(\psi) e^{i(n \alpha-\omega t)} \\
\phi=\phi_{0}(\psi)+\sum_{n} \delta \phi_{n}(\psi) e^{i(n \alpha-\omega t)} \\
P=P_{0}(\psi)+\sum_{n} \delta P_{n}(\psi) e^{i(n \alpha-\omega t)} .
\end{array}\right.
$$

Thus for the density $\rho$, we obtain from Eq. (A1)

$$
\left(\omega-n \omega_{0}(\psi)\right) \delta \rho_{n}=-n \rho_{0}^{\prime}(\psi) \delta \phi_{n}+\frac{3}{2} n \omega_{d 0} \delta P_{n},
$$

and Eq. (A2) is then replaced by

$$
\left(\omega-n \omega_{0}(\psi)\right) \delta P_{n}=-n P_{0}^{\prime}(\psi) \delta \phi_{n}+\frac{2}{3} n \omega_{d 0} \delta M_{2} .
$$

Finally, we write the quasi-neutrality condition (A3) in the following form:

$$
C_{e}\left(\phi_{0}(\psi)-\langle\phi\rangle_{\alpha}\right)-C_{i} \delta_{b}^{2} \phi_{0}^{\prime \prime}(\psi)=\rho_{0}(\psi),
$$

and for the fluctuation potential mode $\delta \phi_{n}$

$$
\left(C_{e}+C_{i} n^{2} \rho_{s}^{2}\right) \delta \phi_{n}-C_{i} \delta_{b}^{2} \delta \phi_{n}^{\prime \prime}=\delta \rho_{n} .
$$

In Eqs. (A5)-(A8), the prime denotes a derivation with respect the variable $\psi$ and we have also introduced the (normalized to $\omega_{d 0}$ ) flow shear in the form $\omega_{0}(\psi)=\phi_{0}^{\prime}(\psi)$. Assuming that the fluctuation term $\delta M_{2}$ can be neglected, and that $\langle\phi\rangle_{\alpha}(\psi, t=0)=\phi_{0}$, a little algebra leads to a second-order equation for the potential fluctuation $\delta \phi_{n}$ in the following form:

$$
\begin{aligned}
& \varphi^{\prime \prime}-\left(k^{2}-\frac{C_{e}}{C_{i} \delta_{b}^{2}} \frac{\omega_{0}-\frac{d\langle\phi\rangle_{\alpha}}{d \psi}}{C-\omega_{0}}-\frac{\omega_{0}^{\prime \prime}}{C-\omega_{0}}+\frac{3}{2} \frac{\omega_{d 0} P_{0}^{\prime}}{C_{i} \delta_{b}^{2}\left(C-\omega_{0}\right)^{2}}\right) \\
& \times \varphi=0,
\end{aligned}
$$

where we have introduced the following notation $C=\frac{\omega}{n}$, $\varphi=\delta \phi_{n}(\psi)$, and $k^{2}=\frac{C_{e}}{C_{i} \delta_{b}^{2}}+\frac{n^{2} \rho_{s}^{2}}{\delta_{b}^{2}}$.

\section{APPENDIX B: THREE-OSCILLATOR MODEL}

The three-oscillator model involves the decay of a pump wave characterized by an interchange type CTIM mode of frequency and toroidal number $\left(\omega_{T}, n_{T}\right)$ into a resonant CTIM mode $\left(\omega_{R}, n_{R}\right)$ and a low-frequency oscillatory zonal flow $\left(\omega_{0}, n_{0}=0\right)$. Here, since the zonal flow is characterized 
by the index 0 with a zero value of the toroidal number and has no dependence in $\alpha$, we impose the condition $n_{T}=n_{R}=n$. The resonant conditions are then given by

$$
\omega_{T}=\omega_{R}+\omega_{0}+\delta \omega \quad \text { and } \quad n_{T}=n_{R}+n_{0},
$$

or equivalently by Eq. (17) where we have also introduced a frequency mismatch of $\delta \omega=\omega_{T}-\omega_{R}-\omega_{0}$. It must be pointed out that in the standard picture of the turbulence, several triads are possible, however, it is here the resonant character of the CTIMs which imposes such a resonant wave-wave interaction. We analyse here the possibility of such a coupling starting from the set of Vlasov equation parameterized by the adiabatic invariants $\kappa$ and $E$. Denoting the distribution $\bar{f}_{\kappa, E}(\psi, \alpha, t)$ by $f$ and assuming that the dissipation is zero $(D(\psi)=0)$ and $J_{0} \phi \simeq \phi$, the Vlasov equation reads as

$$
\frac{\partial f}{\partial t}+\omega_{d}(\kappa) E \frac{\partial f}{\partial \alpha}+[\phi, f]=0
$$

Thus, the parameters $\kappa$ and $E$ have been dropped in notation for simplifying the presentation and we have used the usual notation $\omega_{d}(\kappa)=\omega_{d 0} \bar{\omega}_{d}(\kappa)$, where $\bar{\omega}_{d}$ is the standard dimensionless frequency given by the elliptic functions. We introduce different time and spatial scales in the form

$$
\begin{aligned}
f(\psi, \alpha, t)= & \frac{1}{2} F_{0}(\psi, t) e^{-i \omega_{0} t}+\frac{1}{2} \delta f_{R} e^{i\left(n \alpha-\omega_{R} t\right)} \\
& +\frac{1}{2} \delta f_{T} e^{i\left(n \alpha-\omega_{T} t\right)}+c . c ., \\
\phi(\psi, \alpha, t)= & \frac{1}{2} \phi_{0}(\psi, t) e^{-i \omega_{0} t}+\frac{1}{2} \delta \phi_{R} e^{i\left(n \alpha-\omega_{R} t\right)} \\
& +\frac{1}{2} \delta \phi_{T} e^{i\left(n \alpha-\omega_{T} t\right)}+c . c .
\end{aligned}
$$

where $\delta f_{i}=\delta f_{i}(\psi, \alpha, t)$ and $\delta \phi_{i}=\delta \phi_{i}(\psi, \alpha, t)$ for $i=R, T$ are the slow-varying complex envelopes. We substitute Eqs. (B3) and (B4) into the Vlasov equation (B2) and separate the different scales, following the standard method of research of secular terms. A little algebra leads to a set of nonlinear coupled equations. To obtain the contribution of the zonal flow in terms of slow-varying envelope, we have also introduced an integration over the variable $\alpha$. The equation describing the evolution of the complex zonal flow envelope in the resonant parametric instability is then given by the following equation:

$$
\frac{\partial F_{0}}{\partial t}=-\frac{1}{2}\left\langle\left[\delta \phi_{T}, \delta f_{R}^{*}\right]\right\rangle_{\alpha} e^{-i \delta \omega t}-\frac{1}{2}\left\langle\left[\delta \phi_{R}^{*}, \delta f_{T}\right]\right\rangle_{\alpha} e^{-i \delta \omega t} .
$$

Here $[.,$.$] denotes the standard Poisson Bracket. The energy$ source is here provided by the ion gradient temperature via the interchange mode which acts as a pump wave. Its envelope equation is given by

$$
\frac{\partial \delta f_{T}}{\partial t}+\omega_{d}(\kappa) E \frac{\partial \delta f_{T}}{\partial \alpha}=\frac{1}{2}\left(\frac{\partial F_{0}}{\partial \psi} \frac{\partial \delta \phi_{R}}{\partial \alpha}-\frac{\partial \phi_{0}}{\partial \psi} \frac{\partial \delta f_{R}}{\partial \alpha}\right) e^{i \delta \omega t}
$$

The resonant contribution in term on slow varying (complex) envelope reads as

$$
\frac{\partial \delta f_{R}}{\partial t}+\omega_{d}(\kappa) E \frac{\partial \delta f_{R}}{\partial \alpha}=\frac{1}{2}\left(\frac{\partial F_{0}^{*}}{\partial \psi} \frac{\partial \delta \phi_{T}}{\partial \alpha}-\frac{\partial \phi_{0}^{*}}{\partial \psi} \frac{\partial \delta f_{T}}{\partial \alpha}\right) e^{-i \delta \omega t}
$$

Thus, in Eqs. (B5)-(B7), the labels T, R, and 0 refer, respectively, to the interchange mode, the resonant CTIM mode, and the low-frequency oscillating ZF. The three-wave model, defined by the set of Eqs. (B5)-(B7), has allowed us to obtain the linear conditions for the trapped-ion modes in the (expected) form, i.e., $\omega_{T}=n \omega_{d}(\kappa) E$ and $\omega_{R}=n \omega_{d}(\kappa) E_{r e s}$, while there is no linear counterpart of a dispersion relation for the zonal flow. Indeed, Eq. (B5) has been obtained assuming the following condition:

$$
\omega_{0} F_{0}(\psi, t)=\frac{1}{2} \partial_{\psi}\left\langle\delta \phi_{T} \delta f_{R}^{*}-\delta \phi_{R}^{*} \delta f_{T}\right\rangle_{\alpha}
$$

which indicates that when there is no resonance, the resonant mode is not amplified and $\delta f_{R} \rightarrow \delta f_{T}$ and $\delta \phi_{R} \rightarrow \delta \phi_{T}$. Thus, $\omega_{0} \rightarrow 0$ according to (B8), since in that case, the polarization effects are not taken into account, and we recover the adiabatic condition

$$
\delta f=-\frac{n \delta \phi F_{0}^{\prime}(\psi)}{\omega-n \omega_{d}(\kappa) E-n \phi_{0}^{\prime}(\psi)} .
$$

In Eqs. (B3) and (B4), we have introduced two different scales in the fluctuations: a fast time scale $\omega_{R}$ and $\omega_{T}$ and a slow-time scale $\omega_{0}$. However, we see in Eq. (B8) that the envelope variation is also captured by the $\partial_{\psi}$ derivation acting on a slow scale in poloidal flux coordinate.

${ }^{1}$ K. Itoh, S. I. Itoh, P. H. Diamond, T. S. Hahm, A. Fujisawa, G. R. Tynan, M. Yadi, and Y. Nagashima, Phys. Plasmas 13, 055502 (2006).

${ }^{2}$ P. H. Diamond, AIP Conf. Proc. 1095, 88 (2009).

${ }^{3}$ X. Garbet, Y. Idomura, L. Villard, and T. H. Watanabe, Nucl. Fusion 50, 043002 (2010).

${ }^{4}$ R. Numata, R. Ball, and R. L. Dewar, Phys. Plasmas 14, 102312 (2007).

${ }^{5}$ E. J. Kim and P. H. Diamond, Phys. Plasmas 10, 1698 (2003).

${ }^{6}$ M. A. Malkov, P. H. Diamond, and M. N. Rosenbluth, Phys. Plasmas 8, 5073 (2001).

${ }^{7}$ J. Li and Y. Kishimoto, Phys. Rev. Lett. 89, 115002 (2002).

${ }^{8}$ B. Kadoch, W. J. T. Bos, and K. Schneider, Phys. Rev. Lett. 105, 145001 (2010).

${ }^{9}$ N. Winsor, J. L. Johnson, and J. M. Dawson, Phys. Fluids 11, 2448 (1968).

${ }^{10}$ M. N. Rosenbluth and F. L. Hinton, Phys. Rev. Lett. 80, 724 (1998).

${ }^{11}$ S. V. Novakovskii, C. S. Liu, R. Z. Sagdeev, and M. N. Rosenbluth, Phys. Plasmas 4, 4272 (1997).

${ }^{12}$ P. H. Diamond, S. Champeaux, M. Malkov, A. Das, I. Grunizou, M. N. Rosenbluth, C. Holland, B. Wecht, A. I. Smolyakov, F. L. Hinton, Z. Lin, and T. S. Hahm, Nucl. Fusion 41, 1067 (2001).

${ }^{13}$ G. Darmet, Ph. Ghendrih, Y. Sarazin, X. Garbet, and V. Grandgirard, Commun. Nonlinear Sci. Numer. Simul. 13, 53 (2008).

${ }^{14}$ K. Miki, Y. Kishimoto, N. Miyato, and J. Q. Li, Phys. Rev. Lett. 99, 145003 (2007).

${ }^{15}$ K. Miki, Y. Kishimoto, J. Li, and N. Miyato, Phys. Plasmas 15, 052309 (2008).

${ }^{16}$ K. Miki and P. H. Diamond, Phys. Plasmas 17, 032309 (2010)

${ }^{17}$ B. N. Rogers, W. Dorland, and M. Kotschenreuther, Phys. Rev. Lett. 85, 5336 (2000).

${ }^{18}$ S. Kobayashi and B. N. Rogers, Phys. Plasmas 19, 012315 (2012).

${ }^{19}$ E. J. Kim and P. H. Diamond, Phys. Plasmas 9, 4530 (2002). 
${ }^{20}$ A. Das, A. Sen, S. Mahajan, and P. Kaw, Phys. Plasmas 8, 5104 (2001).

${ }^{21}$ F. Palermo, X. Garbet, A. Ghizzo, T. Cartier-Michaud, P. Ghendrih, V. Grandgirard, and Y. Sarazin, Phys. Plasmas 22, 042304 (2015).

${ }^{22}$ D. K. Gupta, R. J. Fonck, M. R. McKee, D. J. Schlossberg, and M. W. Schafer, Phys. Rev. Lett. 97, 125002 (2006).

${ }^{23}$ G. D. Conway, C. Angioni, F. Ryter, P. Sauter, J. Vicente, and ASDEX Upgrade Team, Phys. Rev. Lett. 106, 065001 (2011).

${ }^{24}$ G. S. Xu, B. N. Wan, H. Q. Wang, H. Y. Guo, H. L. Zhao, A. D. Liu, V. Naulin, P. H. Diamond, G. R. Tynan, M. Xu, R. Chen, M. Jiang, P. Liu, N. Yan, W. Zhang, L. Wang, S. C. Liu, and S. Y. Ding, Phys. Rev. Lett. 107, 125001 (2011).

${ }^{25}$ H. Wang, Y. Todo, and C. C. Kim, Phys. Rev. Lett. 110, 155006 (2013).

${ }^{26}$ I. B. Bernstein, J. M. Greene, and M. D. Kruskal, Phys. Rev. 108, 546 (1957).

${ }^{27}$ D. S. Montgomery, R. J. Focia, H. A. Rose, D. A. Russell, J. A. Cobble, J. C. Fernandez, and R. P. Johnson, Phys. Rev. Lett. 87, 155001 (2001).

${ }^{28}$ T. W. Johnston, Y. Tyshetskiy, A. Ghizzo, and P. Bertrand, Phys. Plasmas 16, 042105 (2009).

${ }^{29}$ A. Ghizzo and D. Del Sarto, Eur. Phys. J. D 68, 275 (2014).

${ }^{30}$ I. Shesterikov, Y. Xu, G. R. Tynan, P. H. Diamond, S. Jachmich, P. Dumortier, M. Vergote, M. Van Schoor, G. Van Oost, and TEXTOR Team, Phys. Rev. Lett. 111, 055006 (2013).
${ }^{31}$ Z. Yan, G. R. McKee, R. Fonck, P. Gohil, R. J. Groebner, and T. H. Osborne, Phys. Rev. Lett. 112, 125002 (2014).

${ }^{32}$ J. Holmboe, Geofys. Publ. 24, 67 (1962).

${ }^{33}$ A. Alexakis, Phys. Fluids 17, 084103 (2005).

${ }^{34}$ S. M. Churikov, Phys. Fluids 23, 114101 (2011).

${ }^{35}$ B. B. Kadomtsev and O. P. Pogutse, Nucl. Fusion 11, 67 (1971).

${ }^{36}$ L. Rayleigh, Proc. London Math. Soc. 11, 57 (1879).

${ }^{37}$ P. G. Drazin and L. N. Howard, "Hydrodynamical stability of parallel flow of inviscid fluid," in Advances in Applied Mechanics, Vol. 17 edited by G. Kuerti (Academic Press, New York 1966), pp. 1-89.

${ }^{38}$ M. M. Shoucri, Int. J. Numer. Methods Eng. 17, 1525 (1981).

${ }^{39}$ W. M. Tang, J. C. Adam, and D. W. Ross, Phys. Fluids 20, 430 (1977).

${ }^{40} \mathrm{H}$. Biglari, P. H. Diamond, and P. W. Terry, Phys. Fluids 31, 2644 (1988).

${ }^{41}$ Y. Kosuga, S. I. Itoh, P. H. Diamond, K. Itoh, and M. Lesur, Phys Plasmas 21, 102303 (2014).

${ }^{42}$ Y. Kosuga and P. H. Diamond, Phys. Plasmas 18, 122305 (2011).

${ }^{43}$ A. Ghizzo, D. Del Sarto, X. Garbet, and Y. Sarazin, Phys. Plasmas 17, 092501 (2010).

${ }^{44}$ A. Ghizzo, M. El Mounden, D. Del Sarto, X. Garbet, and Y. Sarazin, Transp. Theory Stat. Physics 40, 382 (2011).

${ }^{45}$ C. R. Trepte and M. H. Hitchman, Nature 355, 626 (1992).

${ }^{46}$ M. C. McIntyre and T. N. Palmer, J. Atmos. Terr. Phys. 46, 825 (1984). 